\title{
A LEAST-SQUARES TRANSPORT EQUATION COMPATIBLE WITH VOIDS
}

\author{
A Thesis \\ by \\ JON HANSEN
}

\begin{abstract}
Submitted to the Office of Graduate and Professional Studies of Texas A\&M University in partial fulfillment of the requirements for the degree of MASTER OF SCIENCE
\end{abstract}

\begin{abstract}
Chair of Committee, Jim E. Morel
Committee Members, Ryan G. McClarren Gamal Akabani Jean-Luc Guermond

Head of Department, Yassin A. Hassan
\end{abstract}

May 2014

Major Subject: Nuclear Engineering

Copyright 2014 Jon Hansen 


\begin{abstract}
Standard second-order self-adjoint forms of the transport equation, such as the even-parity, odd-parity, and self-adjoint angular flux equation, cannot be used in voids. Perhaps more importantly, they experience numerical convergence difficulties in near-voids. Here we present a new form of a second-order self-adjoint transport equation that has an advantage relative to standard forms in that it can be used in voids or near-voids. Our equation is closely related to the standard least-squares or normal form of the transport equation, which is applicable in a void and has a nonconservative analytic form. However, unlike the standard least-squares form of the transport equation, our least-squares equation is compatible with source iteration. It has been found that the standard least-squares form of the transport equation with a linear-continuous finite-element spatial discretization has difficulty in the thick diffusion limit. Here we extensively test the 1-D slab-geometry version of our scheme with respect to void solutions, spatial convergence rate, and the intermediate and thick diffusion limits. We also define an effective diffusion synthetic acceleration scheme for our discretization. Our conclusion is that our least-squares $S_{n}$ formulation represents an excellent alternative to existing second-order $S_{n}$ transport formulations.
\end{abstract}




\section{ACKNOWLEDGEMENTS}

I would first like to express my gratitude to my advisor Dr. Jim E. Morel for providing invaluable guidance during this project. I would also like to thank Dr. Gamal Akabani, Dr. Jean-Luc Guermond, and Dr. Ryan G. McClarren for serving as committee members. In addition, I would like to thank the Nuclear Regulatory Commission for the graduate fellowship I received during my first year. Finally, I would like to extend special thanks to my family for their love and support. In particular, I would like to say thank you to my loving, encouraging, and patient wife Kelsey whose unwavering support is hugely appreciated. 


\section{TABLE OF CONTENTS}

Page

ABSTRACT ...............................

ACKNOWLEDGEMENTS .......................... iii

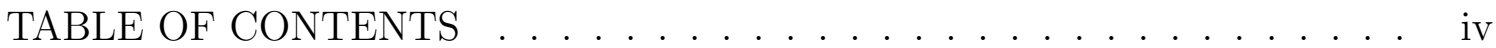

LIST OF FIGURES . . . . . . . . . . . . . . . . . . . . v

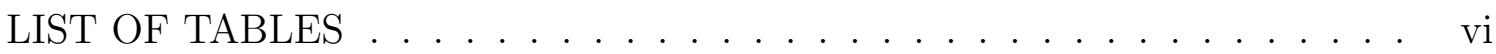

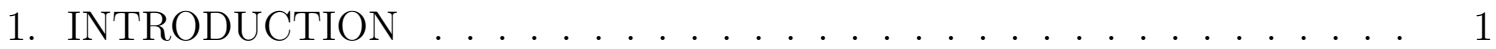

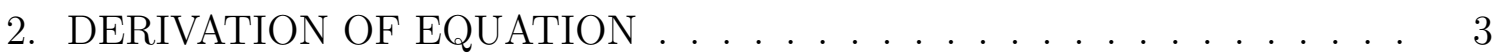

3. DISCRETIZATION OF EQUATION . . . . . . . . . . . . . 7

4. DIFFUSION SYNTHETIC ACCELERATION . . . . . . . . . . . . . 11

5. ASYMPTOTIC DIFFUSION LIMIT . . . . . . . . . . . . . . . . . 14

6. COMPUTATIONAL RESULTS . . . . . . . . . . . . . . . . 23

6.1 Method of Manufactured Solutions . . . . . . . . . . . . . 23

6.2 Compatibility in Void . . . . . . . . . . . . . . . . . 25

6.3 Spectral Radius . . . . . . . . . . . . . . . . . . . . 26

6.4 Rate of Convergence . . . . . . . . . . . . . . . . . . . . 27

6.5 Intermediate Diffusion Limit . . . . . . . . . . . . . . . . . . . 30

6.6 Thick Diffusion Limit . . . . . . . . . . . . . . . . . . . . 33

6.7 Unresolved Boundary Layers . . . . . . . . . . . . . . . . . . . 36

6.8 Interior Boundary Layers . . . . . . . . . . . . . . . . . . . . 39

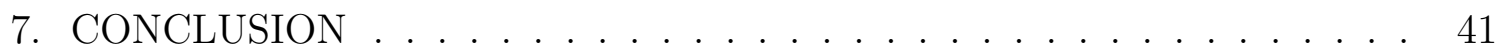

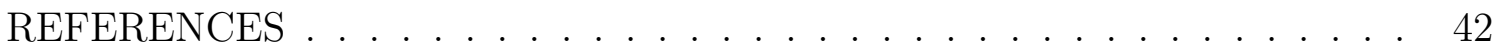




\section{LIST OF FIGURES}

6.1 Numerical $\mathrm{L}_{2}$ error as a function of cell width for the manufactured

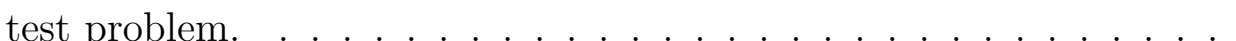

6.2 Scalar flux solution for problem with void. "Analytical" refers to the analytic transport solution. . . . . . . . . . . . . 26

6.3 Spectral radius as a function of cell thickness. . . . . . . . . . . 27

6.4 Numerical error as a function of cell thickness showing second-order spatial convergence for test problems featuring a continuous source

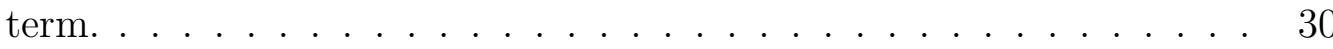

6.5 Numerical error as a function of $\epsilon$ in the intermediate diffusion limit showing first-order accuracy. . . . . . . . . . . . . . . .

6.6 Scalar flux solutions as a function of $\epsilon$ in the thick diffusion limit. "Analytical" refers to the analytic asymptotic diffusion solution. . . . 34

6.7 Numerical error as a function of $\epsilon$ for the intermediate limit test with very thick cells showing approximately first-order accuracy. . . . . . . 36

6.8 Scalar flux solutions for decreasing $\epsilon$ in the thick limit with an incident isotropic boundary flux. "Analytical" refers to the analytic asymptotic diffusion solution. . . . . . . . . . . . . . . . . 38

6.9 Scalar flux solutions for decreasing $\epsilon$ in the thick limit with a unidirectional source normal to the slab showing incorrect behavior within the boundary layer. "Analytical" refers to the analytic asymptotic

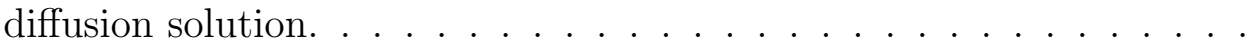

6.10 Scalar flux solutions for decreasing $\epsilon$ in the thick limit with an unresolved interior boundary layer. "Analytical" refers to the analytic asymptotic diffusion solution given void entry with known scalar flux value. . . . . . . . . . . . . . . . . . . . . . 40 40 


\section{LIST OF TABLES}

TABLE

Page

6.1 Numerical error as a function of cell thickness for convergence test problem with an isotropic distributed source. . . . . . . . . . . 29

6.2 Numerical error as a function of cell thickness for convergence test problem with an incident flux at the boundary. . . . . . . . . . . . 29

6.3 Numerical error as a function of $\epsilon$ in the intermediate diffusion limit. 32

6.4 Numerical error as a function of $\epsilon$ for the intermediate limit test with very thick cells. . . . . . . . . . . . . . . 35 


\section{INTRODUCTION}

Standard second-order self-adjoint forms of the transport equation, such as the even-parity, odd-parity [1], and self-adjoint angular flux equation [2], cannot be used in voids. Perhaps more importantly, they experience numerical difficulties in nearvoids [3]. Here we derive a new form of second-order self-adjoint transport equation that has the advantage relative to standard forms that it can be used in voids or near voids. In this paper we extensively study the properties of this equation in 1-D slab geometry. Multidimensional variants of this equation will be considered in a future paper. The only disadvantage of the new equation is that it has a nonconservative analytic form, which means that conservation is only obtained as the numerical solution converges to the analytic solution. We do not consider this to be a major issue since we envision this scheme being used primarily in reactor neutronics calculations in which the variation of the solution is well-resolved by the mesh. Our equation is closely related to the standard least-squares or normal form of the transport equation, which is also applicable in a void and has a non-conservative analytic form. However, our equation is designed to be solved using source iteration, whereas the standard least-squares form is not compatible with source iteration [4]. Manteuffel and Ressel found that the standard least-squares form of the transport equation with linear-continuous finite-element spatial discretization has difficulty in the thick diffusion limit [5]. They were able to eliminate this deficiency by replacing the standard least-squares principle with a weighted least-squares principle. However, the weight function they used is singular in a void, thereby rendering their weighted least-squares equation unsuitable for use in voids. We present computational evidence indicating that our equation with linear-continuous finite-element 
spatial discretization has both the intermediate and thick diffusion limits [6]. Diffusion synthetic acceleration (DSA) can be applied to our equation. However, we do not use a consistently-discretized diffusion equation because the consistent $\mathrm{P}_{1}$ equations derived from our transport equation have a non-standard non-self-adjoint form that cannot be reduced to a simple diffusion equation. Thus we simply apply the same linear-continuous spatial finite-element method to the diffusion equation that we apply to our transport equation and thereby obtain a "partially-consistent" diffusion equation. This diffusion equation yields an unconditionally effective DSA scheme after an ad hoc modification is made at the boundaries to account for non-standard Dirichlet conditions arising from the non-conservative form of our equation.

Although parallel source iteration algorithms for the first-order transport equation on structured meshes have proven to be far more effective for large processor counts than traditionally expected [7], the efficacy of such algorithms for unstructured meshes remains to be demonstrated. Second-order schemes may offer an advantage on unstructured meshes, particularly if multigrid algorithms can be developed for such schemes.

The remainder of this paper is organized as follows. We first derive our equation. Next we show that it can be solved via source iteration with diffusion-synthetic acceleration. A spatial linear-continuous finite-element method is then applied to our equation. The discrete DSA equations are next described. The asymptotic diffusion limit of our transport scheme is then analyzed. We also present computational results demonstrating various properties of our equation, including the ability to treat voids, the effectiveness of our DSA method, the convergence rate of our scheme, the preservation of both the intermediate and thick diffusion limits, and behavior regarding unresolved boundary layers. Finally, we give conclusions and recommendations for future work. 


\section{DERIVATION OF EQUATION}

Let us begin the derivation of our least-squares equation with the first-order monoenergetic transport equation,

$$
\mathbf{L} \psi=\mathbf{S} \psi+\frac{q}{4 \pi}=\frac{\sigma_{s} \phi+q}{4 \pi}=Q
$$

where $\psi\left(\mathrm{n} / \mathrm{cm}^{2}\right.$-s-str $)$ is the angular flux, $\phi\left(\mathrm{n} / \mathrm{cm}^{2}-s\right)$ is the scalar flux defined by $\phi=\int_{4 \pi} \psi d \Omega, q\left(n / \mathrm{cm}^{3}-s\right)$ is the distributed source, and $\mathbf{L}$ is the streaming plus removal operator,

$$
\mathbf{L}=\vec{\Omega} \cdot \vec{\nabla}+\sigma_{t}
$$

$\mathbf{S}$ is the scattering operator,

$$
\mathbf{S}=\frac{\sigma_{s}}{4 \pi} \int_{4 \pi} d \Omega
$$

$\sigma_{t}\left(\mathrm{~cm}^{-1}\right)$ denotes the macroscopic total cross section and $\sigma_{s}\left(\mathrm{~cm}^{-1}\right)$ denotes the macroscopic scattering cross section. Note that isotropic scattering and an isotropic distributed source have been assumed for simplicity without loss of generality. We next define the standard space-angle inner product:

$$
(f, g)=\int_{\mathcal{D}} \int_{4 \pi} f g d \Omega d x .
$$

It is easily shown that the adjoint to $\mathbf{L}$ under this inner product definition is:

$$
\mathbf{L}^{\dagger}=-\vec{\Omega} \cdot \vec{\nabla}+\sigma_{t}
$$


Note that if we treat the direction variable as a parameter and define a purely spatial inner product by eliminating the integration over direction in Eq. (2.4), the same adjoint operator is obtained. We later use this spatial inner product in our derivation of a weak form. Multiplying Eq. (2.1) from the left by $\mathbf{L}^{\dagger}$, we obtain a least-squares or "normal" form of the transport equation that we seek:

$$
\mathbf{L}^{\dagger} \mathbf{L} \psi=\mathbf{L}^{\dagger} Q
$$

This equation can be written using standard notation in the following manner:

$$
-\vec{\Omega} \cdot \vec{\nabla}[\vec{\Omega} \cdot \vec{\nabla} \psi]-\vec{\Omega} \psi \cdot \vec{\nabla} \sigma_{t}+\sigma_{t}^{2} \psi=-\vec{\Omega} \cdot \vec{\nabla} Q+\sigma_{t} Q
$$

Specifically, the equation in 1-D slab-geometry is given by:

$$
-\mu^{2} \frac{\partial^{2} \psi}{\partial x^{2}}-\mu \psi \frac{\partial \sigma_{t}}{\partial x}+\sigma_{t}^{2} \psi=-\mu \frac{\partial Q}{\partial x}+\sigma_{t} Q
$$

Note that in a void this equation reduces to

$$
-\mu^{2} \frac{\partial^{2} \psi}{\partial x^{2}}=0
$$

which is a well-posed equation. Further note that while Eq. (2.8) as a whole is not self-adjoint, the left side of this equation is self-adjoint. The standard least-squares transport equation has the following form,

$$
(\mathbf{L}-\mathbf{S})^{\dagger}(\mathbf{L}-\mathbf{S}) \psi=(\mathbf{L}-\mathbf{S})^{\dagger} Q
$$


which is self-adjoint as a whole. The motivation for our form of the equation is that it is compatible with source iteration. To demonstrate this, we apply source iteration to Eq. (2.6),

$$
\mathbf{L}^{\dagger} \mathbf{L} \psi^{\ell+1}=\mathbf{L}^{\dagger} Q^{\ell}
$$

where $\ell$ is the iteration index. Solving Eq. (2.11) for $\psi^{\ell+1}$, we derive:

$$
\psi^{\ell+1}=\left[\mathbf{L}^{\dagger} \mathbf{L}\right]^{-1} \mathbf{L}^{\dagger} Q^{\ell}=\mathbf{L}^{-1} \mathbf{L}^{\dagger-1} \mathbf{L}^{\dagger} Q^{\ell}=\mathbf{L}^{-1} Q^{\ell}
$$

Note that one analytically obtains the same solution iterate as one obtains by applying source iteration to the standard first-order form of the transport equation. This implies that source iteration (neglecting discretization effects) is identically effective for both the first-order equation and our least-squares equation. Furthermore, it can be seen from Eq. (2.8) that source iteration will require only the solution of a set of independent equations for each direction, which is also the case for the firstorder transport equation. The difference between our equation and the first-order equation is that our equation requires the solution of a second-order self-adjoint equation for each direction while the first-order equation requires the solution of a first-order advection-reaction equation for each direction. In contrast, the standard least-squares equation has coupling between all the directions in the presence of scattering, which makes it incompatible with source iteration.

Because source iteration on our equation is equivalent in terms of solution iterates to source iteration on the first-order equation, it is clear that we can also apply DSA to our equation,

$$
-\mu^{2}{\frac{\partial^{2} \psi^{\ell+1 / 2}}{\partial x^{2}}}^{\ell-\mu \psi^{\ell+1 / 2}} \frac{\partial \sigma_{t}}{\partial x}+\sigma_{t}^{2} \psi^{\ell+1 / 2}=-\mu \frac{\partial Q^{\ell}}{\partial x}+\sigma_{t} Q^{\ell}
$$




$$
\begin{gathered}
-\frac{\partial}{\partial x} \frac{1}{3 \sigma_{t}} \frac{\partial \delta \phi}{\partial x}+\sigma_{a} \delta \phi=\sigma_{s}\left(\phi^{\ell+1 / 2}-\phi^{\ell}\right), \\
\phi^{\ell+1}=\phi^{\ell+1 / 2}+\delta \phi
\end{gathered}
$$

where $\phi\left(n / \mathrm{cm}^{2}-s\right)$ denotes the scalar flux, $\delta \phi\left(n / \mathrm{cm}^{2}-s\right)$ denotes the estimate for the scalar flux iterative error, and the absorption cross section is defined by $\sigma_{a}=\sigma_{t}-\sigma_{s}$ $\left(\mathrm{cm}^{-1}\right)$. 


\section{DISCRETIZATION OF EQUATION}

We discretize our equation using the $\mathrm{S}_{n}$ method in conjunction with a standard piecewise linear-continuous finite-element method. The discrete angular flux unknowns lie at the vertices of the mesh. We use integral indices for vertices and half-integral indices for cell centers. Thus $x_{1}$ is the coordinate of the first vertex, $x_{I}$ is the vertex of the last vertex, and cell $i+1 / 2$ is bounded by $x_{i}$ and $x_{i+1}$. We assume that the cross-sections are constant within each cell, but may vary between cells. Starting from the analytic least-squares transport equation given in Eq. (2.7), we multiply by a test function $\psi^{*}$ and take the spatial inner product. Next, we apply integration by parts to every term containing a spatial derivative to derive the following weak form of the least-squares equation:

$$
\begin{gathered}
\left(\vec{\Omega} \cdot \vec{\nabla} \psi, \vec{\Omega} \cdot \vec{\nabla} \psi^{*}\right)+\left(\sigma_{t} \vec{\Omega} \cdot \vec{\nabla} \psi, \psi^{*}\right)+\left(\sigma_{t} \psi, \vec{\Omega} \cdot \vec{\nabla} \psi^{*}\right)+\left(\sigma_{t}^{2} \psi, \psi^{*}\right)- \\
\left(\frac{q}{4 \pi}, \vec{\Omega} \cdot \vec{\nabla} \psi^{*}\right)-\left(\frac{\sigma_{t} q}{4 \pi}, \psi^{*}\right)-\left(\frac{\sigma_{s} \phi}{4 \pi}, \vec{\Omega} \cdot \vec{\nabla} \psi^{*}\right)-\left(\frac{\sigma_{t} \sigma_{s} \phi}{4 \pi}, \psi^{*}\right)- \\
\left((\vec{n} \cdot \vec{\Omega})\left[\vec{\Omega} \cdot \vec{\nabla} \psi+\sigma_{t} \psi-\frac{q}{4 \pi}-\frac{\sigma_{s} \phi}{4 \pi}\right], \psi^{*}\right)_{\delta \mathcal{D}}=0
\end{gathered}
$$

Since the the least-squares equation satisfies the first-order transport equation on the boundary, the last term of Eq. (3.1) can be set identically to zero. This yields the following weak form of the least-squares equation:

$$
\begin{aligned}
& \left(\vec{\Omega} \cdot \vec{\nabla} \psi, \vec{\Omega} \cdot \vec{\nabla} \psi^{*}\right)+\left(\sigma_{t} \vec{\Omega} \cdot \vec{\nabla} \psi, \psi^{*}\right)+\left(\sigma_{t} \psi, \vec{\Omega} \cdot \vec{\nabla} \psi^{*}\right)+\left(\sigma_{t}^{2} \psi, \psi^{*}\right)- \\
& \left(\frac{q}{4 \pi}, \vec{\Omega} \cdot \vec{\nabla} \psi^{*}\right)-\left(\frac{\sigma_{t} q}{4 \pi}, \psi^{*}\right)-\left(\frac{\sigma_{s} \phi}{4 \pi}, \vec{\Omega} \cdot \vec{\nabla} \psi^{*}\right)-\left(\frac{\sigma_{t} \sigma_{s} \phi}{4 \pi}, \psi^{*}\right)=0
\end{aligned}
$$


Note that with the boundary term set to 0, our least-squares equation using Galerkin methods is identical to least-squares finite element on the first-order transport equation. In this case, both the trial function and the test functions denoted by $\psi^{*}$ are represented in terms of the basis functions defined as follows:

$$
\begin{gathered}
B_{1}(x)=\frac{x_{2}-x}{x_{2}-x_{1}}, \quad \text { for } x \in\left[x_{1}, x_{2}\right], \\
=\quad 0 \quad, \quad \text { otherwise, } \\
B_{i}(x)=\frac{x-x_{i-1}}{x_{i}-x_{i-1}}, \quad \text { for } x \in\left[x_{i-1}, x_{i}\right], i=2, I-1, \\
=\frac{x_{i+1}-x}{x_{i+1}-x_{i}}, \quad \text { for } x \in\left[x_{i}, x_{i+1}\right], i=2, I-1, \\
=\quad 0 \quad \text { otherwise, } \\
B_{I}(x)=\frac{x-x_{I-1}}{x_{I}-x_{I-1}} \quad, \quad \text { for } x \in\left[x_{I-1}, x_{I}\right] \\
=\quad 0 \quad \text { otherwise. }
\end{gathered}
$$

The discrete equation for all $\psi_{i}$ is obtained by expanding the solution in the basis functions, setting the test function to $B_{i}(x)$, and evaluating Eq. (3.2). Since the trial and test spaces are the same, a Galerkin method is obtained. The 1-D interior-mesh 
equation is

$$
\begin{gathered}
-\frac{\mu^{2}}{h_{i+1 / 2}}\left(\psi_{i+1}-\psi_{i}\right)+\frac{\mu^{2}}{h_{i-1 / 2}}\left(\psi_{i}-\psi_{i-1}\right)-\mu \psi_{i}\left(\sigma_{t, i+1 / 2}-\sigma_{t, i-1 / 2}\right)+ \\
\sigma_{t, i+1 / 2}^{2} \frac{h_{i+1 / 2}}{2}\left(\frac{2}{3} \psi_{i}+\frac{1}{3} \psi_{i+1}\right)+\sigma_{t, i-1 / 2}^{2} \frac{h_{i-1 / 2}}{2}\left(\frac{2}{3} \psi_{i}+\frac{1}{3} \psi_{i-1}\right)= \\
-\frac{\mu}{8 \pi}\left[\sigma_{s, i+1 / 2}\left(\phi_{i+1}-\phi_{i}\right)+\sigma_{s, i-1 / 2}\left(\phi_{i}-\phi_{i-1}\right)+q_{i+1}-q_{i-1}\right]-\frac{\mu}{4 \pi} \phi_{i}\left(\sigma_{s, i+1 / 2}-\sigma_{s, i-1 / 2}\right)+ \\
\sigma_{t, i+1 / 2} \frac{h_{i+1 / 2}}{8 \pi}\left[\sigma_{s, i+1 / 2}\left(\frac{2}{3} \phi_{i}+\frac{1}{3} \phi_{i+1}\right)+\left(\frac{2}{3} q_{i}+\frac{1}{3} q_{i+1}\right)\right]+ \\
\sigma_{t, i-1 / 2} \frac{h_{i-1 / 2}}{8 \pi}\left[\sigma_{s, i-1 / 2}\left(\frac{2}{3} \phi_{i}+\frac{1}{3} \phi_{i-1}\right)+\left(\frac{2}{3} q_{i}+\frac{1}{3} q_{i-1}\right)\right]
\end{gathered}
$$

Undergoing the same procedure, the left boundary equation is:

$$
\begin{gathered}
-\frac{\mu^{2}}{h_{3 / 2}}\left(\psi_{2}-\psi_{1}\right)-\mu \sigma_{t, 3 / 2} \psi_{1}+\sigma_{t, 3 / 2}^{2} \frac{h_{3 / 2}}{2}\left(\frac{2}{3} \psi_{1}+\frac{1}{3} \psi_{2}\right)= \\
-\frac{\mu}{8 \pi}\left[\sigma_{s, 3 / 2}\left(\phi_{2}-\phi_{1}\right)+q_{2}-q_{1}\right]-\frac{\mu}{4 \pi}\left(\sigma_{s, 3 / 2} \phi_{1}+q_{1}\right)+ \\
\sigma_{t, 3 / 2} \frac{h_{3 / 2}}{8 \pi}\left[\sigma_{s, 3 / 2}\left(\frac{2}{3} \phi_{1}+\frac{1}{3} \phi_{2}\right)+\left(\frac{2}{3} q_{1}+\frac{1}{3} q_{2}\right)\right], \quad \mu<0 .
\end{gathered}
$$

Equation (3.7) is used only for the outgoing directions. For the incoming directions, we apply a Dirichlet condition, i.e., we simply set $\psi_{1}$ to the boundary flux for incoming values defined by the boundary condition:

$$
\psi_{1}=\psi_{b, L}, \quad \mu>0
$$


An analogous treatment at the right boundary yields:

$$
\begin{gathered}
\frac{\mu^{2}}{h_{I-1 / 2}}\left(\psi_{I}-\psi_{I-1}\right)+\mu \sigma_{t, I-1 / 2} \psi_{I}+\sigma_{t, I-1 / 2}^{2} \frac{h_{I-1 / 2}}{2}\left(\frac{2}{3} \psi_{I}+\frac{1}{3} \psi_{I-1}\right)= \\
-\frac{\mu}{8 \pi}\left[\sigma_{s, I-/ 2}\left(\phi_{I}-\phi_{I-1}\right)+q_{I}-q_{I-1}\right]+\frac{\mu}{4 \pi}\left(\sigma_{s, I-1 / 2} \phi_{I}+q_{I}\right)+ \\
\sigma_{t, I-1 / 2} \frac{h_{I-1 / 2}}{8 \pi}\left[\sigma_{s, I-1 / 2}\left(\frac{2}{3} \phi_{I}+\frac{1}{3} \phi_{I-1}\right)+\left(\frac{2}{3} q_{I}+\frac{1}{3} q_{I-1}\right)\right], \quad \mu>0 .
\end{gathered}
$$

and

$$
\psi_{I}=\psi_{b, R}, \quad \mu<0
$$

Our discrete equations are tridiagonal and the matrix corresponding to the left side of Eq. (2.13a) is symmetric positive-definite. Consequently, we solve our source iteration equations using a direct tridiagonal solver without partial pivoting. 


\section{DIFFUSION SYNTHETIC ACCELERATION}

As previously noted, we can apply diffusion-synthetic acceleration to our leastsquares equation. It is usually desirable to use a discretization of the diffusion equation that is consistent with the discretization of the transport equation. However, because our least-squares equation is not self-adjoint on the whole, it yields consistent analytic $\mathrm{P}_{1}$ equations that are not self-adjoint:

$$
\begin{gathered}
-\frac{1}{3} \frac{\partial^{2} \phi}{\partial x^{2}}-J \frac{\partial \sigma_{t}}{\partial x}+\sigma_{t}^{2} \phi=\sigma_{t}\left(\sigma_{s} \phi+q\right) \\
-\frac{3}{5} \frac{\partial^{2} J}{\partial x^{2}}-\frac{1}{3} \phi \frac{\partial \sigma_{t}}{\partial x}+\sigma_{t}^{2} J=-\frac{1}{3} \frac{\partial}{\partial x}\left(\sigma_{s} \phi+q\right),
\end{gathered}
$$

where $J\left(n / \mathrm{cm}^{2}\right.$-s) denotes the current. Furthermore, and most importantly, we cannot reduce Eqs. (4.1a) and (4.1b) to a single diffusion equation. Thus, we have chosen not to use these equations for DSA purposes. Rather we obtain a "partially" consistent diffusion equation by applying the same finite-element spatial discretization to Eq. (2.13b) that we apply to our least-squares transport equation. The interior mesh diffusion equation is

$$
\begin{gathered}
-\frac{1}{3 \sigma_{t, i+1 / 2} h_{i+1 / 2}}\left(\delta \phi_{i+1}-\delta \phi_{i}\right)+\frac{1}{3 \sigma_{t, i-1 / 2} h_{i-1 / 2}}\left(\delta \phi_{i}-\delta \phi_{i-1}\right)+ \\
\sigma_{a, i+1 / 2} \frac{h_{i+1 / 2}}{2}\left(\frac{2}{3} \delta \phi_{i}+\frac{1}{3} \delta \phi_{i+1}\right)+\sigma_{a, i-1 / 2} \frac{h_{i-1 / 2}}{2}\left(\frac{2}{3} \delta \phi_{i}+\frac{1}{3} \delta \phi_{i-1}\right)= \\
\sigma_{s, i+1 / 2} \frac{h_{i+1 / 2}}{2}\left[\left(\frac{2}{3} \phi_{i}^{\ell+1 / 2}+\frac{1}{3} \phi_{i+1}^{\ell+1 / 2}\right)-\left(\frac{2}{3} \phi_{i}^{\ell}+\frac{1}{3} \phi_{i+1}^{\ell}\right)\right]+ \\
\sigma_{s, i-1 / 2} \frac{h_{i-1 / 2}}{2}\left[\left(\frac{2}{3} \phi_{i}^{\ell+1 / 2}+\frac{1}{3} \phi_{i-1}^{\ell+1 / 2}\right)-\left(\frac{2}{3} \phi_{i}^{\ell}+\frac{1}{3} \phi_{i-1}^{\ell}\right)\right] .
\end{gathered}
$$


The interior mesh update equation is

$$
\phi_{i}^{\ell+1}=\phi_{i}^{\ell+1 / 2}+\delta \phi_{i}, \quad i=2, I-1 .
$$

The left boundary equation (assuming source or vacuum boundary conditions for the transport equation) that was initially used was

$$
\begin{gathered}
-\frac{1}{3 \sigma_{t, 3 / 2} h_{3 / 2}}\left(\delta \phi_{2}-\delta \phi_{1}\right)+\sigma_{a, 3 / 2} \frac{h_{3 / 2}}{2}\left(\frac{2}{3} \delta \phi_{1}+\frac{1}{3} \delta \phi_{2}\right)= \\
\sigma_{s, 3 / 2} \frac{h_{3 / 2}}{2}\left[\left(\frac{2}{3} \phi_{1}^{\ell+1 / 2}+\frac{1}{3} \phi_{2}^{\ell+1 / 2}\right)-\left(\frac{2}{3} \phi_{1}^{\ell}+\frac{1}{3} \phi_{2}^{\ell}\right)\right] .
\end{gathered}
$$

The left boundary update equation is

$$
\phi_{1}^{\ell+1}=\phi_{1}^{\ell+1 / 2}+\frac{7}{8} \delta \phi_{1}
$$

the factor of $7 / 8$ comes from a detailed analysis of the diffusion boundary conditions that is usually neglected. However, replacing it with a factor of one has a minimal effect. During initial testing we discovered that this treatment at the boundaries lead to instability for highly diffusive problems with optically-thick cells. We speculate that this difficulty is related to the use of Dirichlet transport boundary conditions since only half the directions at a boundary actually satisfy the transport equation. We were able to eliminate the instability and obtain a very good overall DSA scheme by setting the residual to zero in the boundary equations. This results in the following equation for the left boundary:

$$
-\frac{1}{3 \sigma_{t, 3 / 2} h_{3 / 2}}\left(\delta \phi_{2}-\delta \phi_{1}\right)+\sigma_{a, 3 / 2} \frac{h_{3 / 2}}{2}\left(\frac{2}{3} \delta \phi_{1}+\frac{1}{3} \delta \phi_{2}\right)=0
$$


Equation (4.5) is retained for the update. An analogous treatment results in the following equation for the right boundary:

$$
\frac{1}{3 \sigma_{t, I-1 / 2} h_{I-1 / 2}}\left(\delta \phi_{I}-\delta \phi_{I-1}\right)+\sigma_{a, I-1 / 2} \frac{h_{I-1 / 2}}{2}\left(\frac{2}{3} \delta \phi_{I}+\frac{1}{3} \delta \phi_{I-1}\right)=0
$$

with the following update equation:

$$
\phi_{I}^{\ell+1}=\phi_{I}^{\ell+1 / 2}+\frac{7}{8} \delta \phi_{I}
$$

The discrete diffusion matrix associated with our DSA scheme is symmetric positive-definite. Hence we solve this equation using the same tridiagonal solver used to solve our source iteration equations. Note that the DSA scheme in its current form cannot be formally implemented within a material void, since the total interaction cross section appears in the denominator of Eq. (4.2) and the corresponding boundary expressions. However, we define a minimum value of $\sigma_{t}$ for purposes of defining the diffusion coefficients in the DSA equations. When the physical cross section is less than then minimum cross section, the minimum value is used. Otherwise, the physical value is used. This minimum value was set to $10^{-3} \mathrm{~cm}^{-1}$ for the calculations performed in this paper. 


\section{ASYMPTOTIC DIFFUSION LIMIT}

Next, we explore the asymptotic diffusion limit of our transport scheme. For a linear transport problem, the diffusion equation can be derived using an asymptotic expansion $[8,9]$. First, we show that the analytic form of our proposed transport equation retains the asymptotic properties of the standard first-order equation in the diffusion limit. This must be the case, since our transport equation and the standard first-order form of the transport equation must yield the same analytic solutions. Nonetheless, it is useful to show the asymptotic analysis for the analytic equation, since this will provide guidance for similar analysis done for the discretized form. Next, we numerically show that our 1-D discretized equation preserves both the intermediate and thick diffusion limits [6]. We also present an asymptotic analysis for the thick limit.

An analytic asymptotic diffusion limit analysis is performed for our proposed form of the transport equation by first scaling certain terms in the transport equation using a small parameter, $\epsilon$. The transport solution to the scaled equations is then expanded in a power series in $\epsilon$. Finally, a heirarchical set of equations for the expansion coefficients is obtained by equating all terms multiplying each power of $\epsilon$.

The asymptotic limit is achieved as $\epsilon \rightarrow 0$. The scaling for the diffusion limit follows:

$$
\begin{gathered}
\sigma_{t} \rightarrow \frac{\sigma_{t}}{\epsilon}, \\
\sigma_{a} \rightarrow \sigma_{a} \epsilon, \\
\sigma_{s} \rightarrow \frac{\sigma_{t}}{\epsilon}-\sigma_{a} \epsilon, \\
q \rightarrow q \epsilon .
\end{gathered}
$$


Thus, in the diffusion limit the total cross section becomes infinite, the absorption cross section goes to zero, and the distributed source goes to zero. The cross section scalings physically characterize the diffusion limit, but the source scaling simply ensures that the solution remains of size $O(1)$ as $\epsilon \rightarrow 0[8]$.

As a reminder, the analytic form of our least-squares transport equation in 1-D slab geometry is given by:

$$
-\mu^{2} \frac{\partial^{2} \psi}{\partial x^{2}}-\mu \psi \frac{\partial \sigma_{t}}{\partial x}+\sigma_{t}^{2} \psi=-\mu \frac{\partial Q}{\partial x}+\sigma_{t} Q
$$

We represent the source term $Q$ in terms of isotropic scattering and an isotropic inhomogeneous source:

$$
Q=\frac{\sigma_{s} \phi+q}{4 \pi} .
$$

Using the substitutions proposed above, we define an asymptotic form of the least-squares transport equation in 1-D slab geometry:

$$
\begin{gathered}
-\mu^{2} \frac{\partial^{2} \psi}{\partial x^{2}}-\mu \psi \frac{1}{\epsilon} \frac{\partial \sigma_{t}}{\partial x}+\frac{\sigma_{t}^{2}}{\epsilon^{2}} \psi= \\
-\frac{\mu}{4 \pi} \frac{\partial}{\partial x}\left[\left(\frac{\sigma_{t}}{\epsilon}-\sigma_{a} \epsilon\right) \phi+q \epsilon\right]+\frac{1}{4 \pi} \frac{\sigma_{t}}{\epsilon}\left[\left(\frac{\sigma_{t}}{\epsilon}-\sigma_{a} \epsilon\right) \phi+q \epsilon\right] .
\end{gathered}
$$

Next, we multiply through Eq. (5.4) by a factor of $\epsilon^{2}$ and proceed to group terms. The asymptotic form of the transport equation becomes: 


$$
\begin{gathered}
-\epsilon^{2} \mu^{2} \frac{\partial^{2} \psi}{\partial x^{2}}-\epsilon \mu \psi \frac{\partial \sigma_{t}}{\partial x}+\sigma_{t}^{2} \psi= \\
-\epsilon \frac{\mu}{4 \pi} \frac{\partial}{\partial x}\left[\left(\sigma_{t}-\sigma_{a} \epsilon^{2}\right) \phi+q \epsilon^{2}\right]+\frac{1}{4 \pi} \sigma_{t}\left[\left(\sigma_{t}-\sigma_{a} \epsilon^{2}\right) \phi+q \epsilon^{2}\right] .
\end{gathered}
$$

Now assume an asymptotic expansion of the angular flux:

$$
\psi=\sum_{n=1}^{\infty} \psi^{(n)} \epsilon^{n}
$$

Quantities with a superscript $(n)$ are called $n$ th-order quantities. Gathering the zeroth-order terms from Eq. (5.5) and simplifying the expression, we can write the zeroth-order asymptotic equation as:

$$
\psi^{(0)}=\frac{1}{4 \pi} \phi^{(0)}
$$

Equation (5.7) represents the expected result. Namely, the leading-order angular flux is isotropic.

Gathering the first-order terms from Eq. (5.5), we obtain the first-order equation:

$$
-\mu \psi^{(0)} \frac{\partial \sigma_{t}}{\partial x}+\sigma_{t}^{2} \psi^{(1)}=-\frac{\mu}{4 \pi} \frac{\partial}{\partial x}\left(\sigma_{t} \phi^{(0)}\right)+\frac{1}{4 \pi} \sigma_{t}^{2} \phi^{(1)}
$$

Next, we multiply Eq. (5.8) by $\mu$ and proceed to integrate over all directions. This results in the following relation:

$$
\sigma_{t}^{2} J^{(1)}=-\frac{1}{4 \pi} \sigma_{t} \frac{\partial \phi^{(0)}}{\partial x} \int_{0}^{2 \pi} d \theta \int_{-1}^{1} d \mu \mu^{2}+\frac{1}{4 \pi} \sigma_{t}^{2} \phi^{(1)} \int_{0}^{2 \pi} d \theta \int_{-1}^{1} d \mu \mu
$$

Upon evaluating the integrals over all directions, we obtain the first-order current: 


$$
J^{(1)}=-\frac{1}{3 \sigma_{t}} \frac{\partial \phi^{(0)}}{\partial x}
$$

Once again, this is the expected analytic result for the first-order asymptotic equation. The equation represents Fick's Law for the 1-D slab-geometry treated in this problem.

Finally, we gather $\epsilon^{2}$ terms to write the second-order equation for the asymptotic analysis:

$$
\begin{gathered}
-\mu^{2} \frac{\partial^{2} \psi^{(0)}}{\partial x^{2}}-\mu \psi^{(1)} \frac{\partial \sigma_{t}}{\partial x}+\sigma_{t}^{2} \psi^{(2)}= \\
-\frac{1}{4 \pi} \sigma_{t} \sigma_{a} \phi^{(0)}+\frac{1}{4 \pi} \sigma_{t} q-\frac{\mu}{4 \pi} \frac{\partial}{\partial x}\left(\sigma_{t} \phi^{(1)}\right)+\frac{1}{4 \pi} \sigma_{t}^{2} \phi^{(2)} .
\end{gathered}
$$

Substituting Eq. (5.7) into Eq. (5.11), we derive the following form of the 2ndorder equation:

$$
\begin{gathered}
-\frac{\mu^{2}}{4 \pi} \frac{\partial^{2} \phi^{(0)}}{\partial x^{2}}-\mu \psi^{(1)} \frac{\partial \sigma_{t}}{\partial x}+\frac{1}{4 \pi} \sigma_{t}^{2} \phi^{(2)}= \\
-\frac{1}{4 \pi} \sigma_{t} \sigma_{a} \phi^{(0)}+\frac{1}{4 \pi} \sigma_{t} q-\frac{\mu}{4 \pi} \frac{\partial}{\partial x}\left(\sigma_{t} \phi^{(1)}\right)+\frac{1}{4 \pi} \sigma_{t}^{2} \phi^{(2)} .
\end{gathered}
$$

Next, we integrate Eq. (5.12) over all directions to yield the following form:

$$
\begin{gathered}
-\frac{1}{3} \frac{\partial^{2} \phi^{(0)}}{\partial x^{2}}-J^{(1)} \frac{\partial \sigma_{t}}{\partial x}+\sigma_{t}^{2} \phi^{(2)}= \\
-\sigma_{t} \sigma_{a} \phi^{(0)}+\sigma_{t} q+\sigma_{t}^{2} \phi^{(2)} .
\end{gathered}
$$

By substituting Eq. (5.10) into Eq. (5.13) and dividing both sides by $\sigma_{t}$, we obtain 
the desired diffusion equation for the leading-order scalar flux:

$$
-\frac{\partial}{\partial x}\left(\frac{1}{3 \sigma_{t}} \frac{\partial \phi^{(0)}}{\partial x}\right)+\sigma_{a} \phi^{(0)}=q .
$$

Thus, we have shown that the proposed least-squares transport equation retains the asymptotic properties of the standard first-order equation in the diffusion limit.

Next, we explore the asymptotic diffusion limit for the discretized form of the least-squares transport equation. In numerical analysis, there are two types of asymptotic diffusion limits that are useful to analyze: the intermediate diffusion limit and the thick diffusion limit [6]. Under both regimes, transport parameters are varied by a positive scaling factor $\epsilon$ in the same manner described in Eq. (5.1a) through Eq. (5.1d). The difference between the intermediate and thick diffusion limit lies in the treatment of the cell width $h$. In the intermediate diffusion limit, the cell width $h$ is scaled by $\epsilon$. Since $\sigma_{t}$ is divided by $\epsilon$, the total number of mean free paths per cell is constant in the intermediate diffusion limit. For the thick diffusion limit, the cell width $h$ is not dependent upon $\epsilon$, so the number of mean free paths per cell approaches infinity as $\epsilon \rightarrow 0$. We computationally investigate both the intermediate and thick limits, and the thick limit is considered theoretically.

Lumping of the removal and source terms greatly simplifies the analysis. Thus, we start with the discretized form of the least-squares transport equation on the interior-mesh and lump these terms to obtain: 


$$
\begin{gathered}
-\frac{\mu^{2}}{h_{i+1 / 2}}\left(\psi_{i+1}-\psi_{i}\right)+\frac{\mu^{2}}{h_{i-1 / 2}}\left(\psi_{i}-\psi_{i-1}\right)-\mu \psi_{i}\left(\sigma_{t, i+1 / 2}-\sigma_{t, i-1 / 2}\right)+ \\
\sigma_{t, i+1 / 2}^{2} \frac{h_{i+1 / 2}}{2} \psi_{i}+\sigma_{t, i-1 / 2}^{2} \frac{h_{i-1 / 2}}{2} \psi_{i}= \\
-\frac{\mu}{8 \pi}\left[\sigma_{s, i+1 / 2}\left(\phi_{i+1}-\phi_{i}\right)+\sigma_{s, i-1 / 2}\left(\phi_{i}-\phi_{i-1}\right)+q_{i+1}-q_{i-1}\right]-\frac{\mu}{4 \pi} \phi_{i}\left(\sigma_{s, i+1 / 2}-\sigma_{s, i-1 / 2}\right)+ \\
\sigma_{t, i+1 / 2} \frac{h_{i+1 / 2}}{8 \pi}\left[\sigma_{s, i+1 / 2} \phi_{i}+q_{i}\right]+ \\
\sigma_{t, i-1 / 2} \frac{h_{i-1 / 2}}{8 \pi}\left[\sigma_{s, i-1 / 2} \phi_{i}+q_{i}\right]
\end{gathered}
$$

Upon making the substitutions outlined in Eq. (5.1a) through Eq. (5.1d) and multiplying through both sides of the equation by a factor of $\epsilon^{2}$, we derive the following form of the asymptotic equation for the discretized case:

$$
\begin{gathered}
-\epsilon^{2} \frac{\mu^{2}}{h_{i+1 / 2}}\left(\psi_{i+1}-\psi_{i}\right)+\epsilon^{2} \frac{\mu^{2}}{h_{i-1 / 2}}\left(\psi_{i}-\psi_{i-1}\right)-\epsilon \mu \psi_{i}\left(\sigma_{t, i+1 / 2}-\sigma_{t, i-1 / 2}\right)+ \\
\sigma_{t, i+1 / 2}^{2} \frac{h_{i+1 / 2}}{2} \psi_{i}+\sigma_{t, i-1 / 2}^{2} \frac{h_{i-1 / 2}}{2} \psi_{i}= \\
-\epsilon \frac{\mu}{8 \pi}\left[\left(\sigma_{t, i+1 / 2}-\sigma_{a, i+1 / 2} \epsilon^{2}\right)\left(\phi_{i+1}-\phi_{i}\right)+\left(\sigma_{t, i-1 / 2}-\sigma_{a, i-1 / 2} \epsilon^{2}\right)\left(\phi_{i}-\phi_{i-1}\right)+q_{i+1} \epsilon^{2}-q_{i-1} \epsilon^{2}\right]- \\
\epsilon \frac{\mu}{4 \pi} \phi_{i}\left[\left(\sigma_{t, i+1 / 2}-\sigma_{a, i+1 / 2} \epsilon^{2}\right)-\left(\sigma_{t, i-1 / 2}-\sigma_{a, i-1 / 2} \epsilon^{2}\right)\right]+ \\
\sigma_{t, i+1 / 2} \frac{h_{i+1 / 2}}{8 \pi}\left[\left(\sigma_{t, i+1 / 2}-\sigma_{a, i+1 / 2} \epsilon^{2}\right) \phi_{i}+q_{i} \epsilon^{2}\right]+ \\
\sigma_{t, i-1 / 2} \frac{h_{i-1 / 2}}{8 \pi}\left[\left(\sigma_{t, i-1 / 2}-\sigma_{a, i-1 / 2} \epsilon^{2}\right) \phi_{i}+q_{i} \epsilon^{2}\right]
\end{gathered}
$$

From the asymptotic equation appearing in Eq. (5.16), we gather $\epsilon^{0}$ terms to form the following zeroth-order equation:

$$
\sigma_{t, i+1 / 2}^{2} \frac{h_{i+1 / 2}}{2} \psi_{i}^{(0)}+\sigma_{t, i-1 / 2}^{2} \frac{h_{i-1 / 2}}{2} \psi_{i}^{(0)}=\sigma_{t, i+1 / 2}^{2} \frac{h_{i+1 / 2}}{8 \pi} \phi_{i}^{(0)}+\sigma_{t, i-1 / 2}^{2} \frac{h_{i-1 / 2}}{8 \pi} \phi_{i}^{(0)} .
$$


The equation above is further reduced to:

$$
\psi_{i}^{(0)}=\frac{1}{4 \pi} \phi_{i}^{(0)}
$$

This result is consistent with the the analytic case, since the leading-order angular flux is isotropic.

Next, we gather $\epsilon^{1}$ terms from Eq. (5.16) to form the first-order asymptotic equation:

$$
\begin{gathered}
-\mu \psi_{i}^{(0)}\left(\sigma_{t, i+1 / 2}-\sigma_{t, i-1 / 2}\right)+\sigma_{t, i+1 / 2}^{2} \frac{h_{i+1 / 2}}{2} \psi_{i}^{(1)}+\sigma_{t, i-1 / 2}^{2} \frac{h_{i-1 / 2}}{2} \psi_{i}^{(1)}= \\
-\frac{\mu}{8 \pi}\left[\sigma_{t, i+1 / 2}\left(\phi_{i+1}^{(0)}-\phi_{i}^{(0)}\right)+\sigma_{t, i-1 / 2}\left(\phi_{i}^{(0)}-\phi_{i-1}^{(0)}\right)\right]-\frac{\mu}{4 \pi} \phi_{i}^{(0)}\left(\sigma_{t, i+1 / 2}-\sigma_{t, i-1 / 2}\right)+ \\
\sigma_{t, i+1 / 2}^{2} \frac{h_{i+1 / 2}}{8 \pi} \phi_{i}^{(1)}+\sigma_{t, i-1 / 2}^{2} \frac{h_{i-1 / 2}}{8 \pi} \phi_{i}^{(1)}
\end{gathered}
$$

Next, we multiply both sides of Eq. (5.19) by $\mu$, integrate over all directions, and solve for the current to yield the relation:

$$
J_{i}^{(1)}=-\frac{1}{3} \frac{\sigma_{t, i+1 / 2}\left(\phi_{i+1}^{(0)}-\phi_{i}^{(0)}\right)+\sigma_{t, i-1 / 2}\left(\phi_{i}^{(0)}-\phi_{i-1}^{(0)}\right)}{\sigma_{t, i+1 / 2}^{2} h_{i+1 / 2}+\sigma_{t, i-1 / 2}^{2} h_{i-1 / 2}}
$$

Although it resembles Fick's law, it is not clear that this expression is consistent with the analytic result.

Finally, we evaluate the second-order asymptotic equation for the discretized case 
by gathering $\epsilon^{2}$ terms from both sides of Eq. (5.16):

$$
\begin{gathered}
-\frac{\mu^{2}}{h_{i+1 / 2}}\left(\psi_{i+1}^{(0)}-\psi_{i}^{(0)}\right)+\frac{\mu^{2}}{h_{i-1 / 2}}\left(\psi_{i}^{(0)}-\psi_{i-1}^{(0)}\right)-\mu \psi_{i}^{(1)}\left(\sigma_{t, i+1 / 2}-\sigma_{t, i-1 / 2}\right)+ \\
\sigma_{t, i+1 / 2}^{2} \frac{h_{i+1 / 2}}{2} \psi_{i}^{(2)}+\sigma_{t, i-1 / 2}^{2} \frac{h_{i-1 / 2}}{2} \psi_{i}^{(2)}= \\
-\frac{\mu}{8 \pi}\left[\sigma_{t, i+1 / 2}\left(\phi_{i+1}^{(1)}-\phi_{i}^{(1)}\right)+\sigma_{t, i-1 / 2}\left(\phi_{i}^{(1)}-\phi_{i-1}^{(1)}\right)\right]-\frac{\mu}{4 \pi} \phi_{i}^{(1)}\left(\sigma_{t, i+1 / 2}-\sigma_{t, i-1 / 2}\right)+ \\
\sigma_{t, i+1 / 2}^{2} \frac{h_{i+1 / 2}}{8 \pi} \phi_{i}^{(2)}+\sigma_{t, i-1 / 2}^{2} \frac{h_{i-1 / 2}}{8 \pi} \phi_{i}^{(2)}- \\
\frac{1}{8 \pi} h_{i+1 / 2} \sigma_{t, i+1 / 2} \sigma_{a, i+1 / 2} \phi_{i}^{(0)}+\frac{1}{8 \pi} h_{i+1 / 2} \sigma_{t, i+1 / 2} q_{i}- \\
\frac{1}{8 \pi} h_{i-1 / 2} \sigma_{t, i-1 / 2} \sigma_{a, i-1 / 2} \phi_{i}^{(0)}+\frac{1}{8 \pi} h_{i-1 / 2} \sigma_{t, i-1 / 2} q_{i}
\end{gathered}
$$

Next, we substitute Eq. (5.18) into Eq. (5.21). This leads to the following form of the second-order asymptotic equation:

$$
\begin{gathered}
-\frac{\mu^{2}}{4 \pi} \frac{1}{h_{i+1 / 2}}\left(\phi_{i+1}^{(0)}-\phi_{i}^{(0)}\right)+\frac{\mu^{2}}{4 \pi} \frac{1}{h_{i-1 / 2}}\left(\phi_{i}^{(0)}-\phi_{i-1}^{(0)}\right)-\mu \psi_{i}^{(1)}\left(\sigma_{t, i+1 / 2}-\sigma_{t, i-1 / 2}\right)+ \\
\frac{\phi_{i}^{(2)}}{4 \pi}\left[\frac{\sigma_{t, i+1 / 2}^{2} h_{i+1 / 2}}{2}+\frac{\sigma_{t, i-1 / 2}^{2} h_{i-1 / 2}}{2}\right]= \\
-\frac{\mu}{8 \pi}\left[\sigma_{t, i+1 / 2}\left(\phi_{i+1}^{(1)}-\phi_{i}^{(1)}\right)+\sigma_{t, i-1 / 2}\left(\phi_{i}^{(1)}-\phi_{i-1}^{(1)}\right)\right]-\frac{\mu}{4 \pi} \phi_{i}^{(1)}\left(\sigma_{t, i+1 / 2}-\sigma_{t, i-1 / 2}\right)+ \\
\frac{\phi_{i}^{(2)}}{4 \pi}\left[\frac{\sigma_{t, i+1 / 2}^{2} h_{i+1 / 2}}{2}+\frac{\sigma_{t, i-1 / 2}^{2} h_{i-1 / 2}}{2}\right]- \\
\frac{\phi_{i}^{(0)}}{4 \pi}\left[\frac{\sigma_{t, i+1 / 2} \sigma_{a, i+1 / 2} h_{i+1 / 2}}{2}+\frac{\sigma_{t, i-1 / 2} \sigma_{a, i-1 / 2} h_{i-1 / 2}}{2}\right]+ \\
\frac{q_{i}^{(2)}}{4 \pi}\left[\frac{\sigma_{t, i+1 / 2} h_{i+1 / 2}}{2}+\frac{\sigma_{t, i-1 / 2} h_{i-1 / 2}}{2}\right]
\end{gathered}
$$

Finally, we integrate Eq. (5.22) over all directions and substitute Eq. (5.20) to form the final reduced form of the second-order equation for the discretized case: 


$$
\begin{gathered}
-\frac{1}{3} \frac{1}{h_{i+1 / 2}}\left(\phi_{i+1}^{(0)}-\phi_{i}^{(0)}\right)+\frac{1}{3} \frac{1}{h_{i-1 / 2}}\left(\phi_{i}^{(0)}-\phi_{i-1}^{(0)}\right)+ \\
\frac{1}{3}\left(\sigma_{t, i+1 / 2}-\sigma_{t, i-1 / 2}\right)\left[\frac{\sigma_{t, i+1 / 2}\left(\phi_{i+1}^{(0)}-\phi_{i}^{(0)}\right)+\sigma_{t, i-1 / 2}\left(\phi_{i}^{(0)}-\phi_{i-1}^{(0)}\right)}{\sigma_{t, i+1 / 2}^{2} h_{i+1 / 2}+\sigma_{t, i-1 / 2}^{2} h_{i-1 / 2}}\right]= \\
-\phi_{i}^{(0)}\left[\frac{\sigma_{t, i+1 / 2} \sigma_{a, i+1 / 2} h_{i+1 / 2}}{2}+\frac{\sigma_{t, i-1 / 2} \sigma_{a, i-1 / 2} h_{i-1 / 2}}{2}\right]+ \\
q_{i}\left[\frac{\sigma_{t, i+1 / 2} h_{i+1 / 2}}{2}+\frac{\sigma_{t, i-1 / 2} h_{i-1 / 2}}{2}\right]
\end{gathered}
$$

Equation (5.23) closely resembles Eq. (5.14) from the analytic case. It is clear that Equation (5.23) is valid when the material cross sections are homogeneous, because it reduces to the standard lumped linear-continuous FEM diffusion discretization:

$$
\begin{gathered}
-\frac{1}{3 \sigma_{t}} \frac{1}{h_{i+1 / 2}}\left(\phi_{i+1}^{(0)}-\phi_{i}^{(0)}\right)+\frac{1}{3 \sigma_{t}} \frac{1}{h_{i-1 / 2}}\left(\phi_{i}^{(0)}-\phi_{i-1}^{(0)}\right)+ \\
-\phi_{i}^{(0)}\left[\frac{\sigma_{a} h_{i+1 / 2}}{2}+\frac{\sigma_{a} h_{i-1 / 2}}{2}\right]+ \\
q_{i}\left[\frac{\sigma_{t} h_{i+1 / 2}}{2}+\frac{\sigma_{t} h_{i-1 / 2}}{2}\right]
\end{gathered}
$$

For the general case with inhomogeneous cross sections, however, it is not obvious that Eq. (5.23) is valid, i.e., that it is a convergent diffusion discretization. Thus, we choose to perform numerical experiments using the method of manufactured solutions to show that the discretized equation is in fact a convergent diffusion discretization for an inhomogeneous test case. The analysis is shown in the following section. 


\section{COMPUTATIONAL RESULTS}

In this section, we present computational results that demonstrate various properties of our scheme. The results were obtained using a code written with MATLAB [10]. All calculations described up to Section 6.4 were performed using source iteration or DSA when specified using a relative pointwise convergence tolerance for scalar flux equal to $10^{-4}$. All calculations described in Section 6.4 or after were performed using the GMRES Krylov method preconditioned with DSA. The Krylov iterations were terminated when the $L_{2}$ norm of the residual divided by the $L_{2}$ norm of the source vector was less than a value of $10^{-10}$.

\subsection{Method of Manufactured Solutions}

We first explore the whether Eq. (5.24) represents a valid consistent diffusion discretization for a problem with inhomogeneous cross sections. To test the scheme, the method of manufactured solutions is utilized. An arbitrary analytic form is first assumed for the scalar flux and then substituted into the analytic diffusion equation. The assumed scalar flux is then made to satisfy the diffusion equation by defining the inhomogeneous source as follows:

$$
q=-\frac{\partial}{\partial x}\left(D \frac{\partial \phi}{\partial x}\right)+\sigma_{a} \phi
$$

The manufactured discrete distributed source $q_{i}$ that appears in Eq. (5.23) is obtained simply by evaluating Eq. (6.1) at the mesh vertices:

$$
q_{i}=\left.\left[-\frac{\partial}{\partial x}\left(D \frac{\partial \phi}{\partial x}\right)+\sigma_{a} \phi\right]\right|_{x=x_{i}}
$$


To obtain a manufactured test for a problem with inhomogeneous material properties, we assume a total interaction cross section that varies with position according to $\sigma_{t}(x)=\sin \left(\frac{\pi x}{R}\right)+0.5$ where $R=2.0 \mathrm{~cm}$ represents the width of the slab. A pure absorbing medium is considered where $\sigma_{a}=\sigma_{t}$ without loss of generality. Furthermore, we assume the following form for the scalar flux: $\phi(x)=\sin \left(\frac{\pi x}{R}\right)$. Therefore, Eq. (6.2) becomes:

$q_{i}=\frac{4 \pi}{3} \frac{1}{\sigma_{t}\left(x_{i}\right)}\left(\frac{\pi}{R}\right)^{2} \sin \left(\frac{\pi x_{i}}{R}\right)+\frac{4 \pi}{3} \frac{1}{\sigma_{t}^{2}\left(x_{i}\right)}\left(\frac{\pi}{R}\right)^{2} \cos ^{2}\left(\frac{\pi x_{i}}{R}\right)+4 \pi \sigma_{a}\left(x_{i}\right) \sin \left(\frac{\pi x_{i}}{R}\right)$.

Next the expression for $q_{i}$ from Eq. (6.3) is substituted into Eq. (5.23), and we proceed to solve for $\phi_{i}^{(0)}$ while setting the numerical solution to zero at the left and right boundary vertices. If Eq. (5.23) is a valid form of diffusion discretization, we expect to find that the numerical flux distribution $\phi_{i}^{(0)}$ converges to $\phi\left(x_{i}\right)=\sin \left(\frac{\pi x_{i}}{R}\right)$ at each $x_{i}$. Indeed, Fig. 6.1 shows on a log-log plot that the numerical solution is converging to the exact solution with second-order accuracy. This numerical result suggests that Eq. (5.23) represents a valid diffusion discretization for the general inhomogeneous case. Further computational evidence presented later in the section supports this statement. 


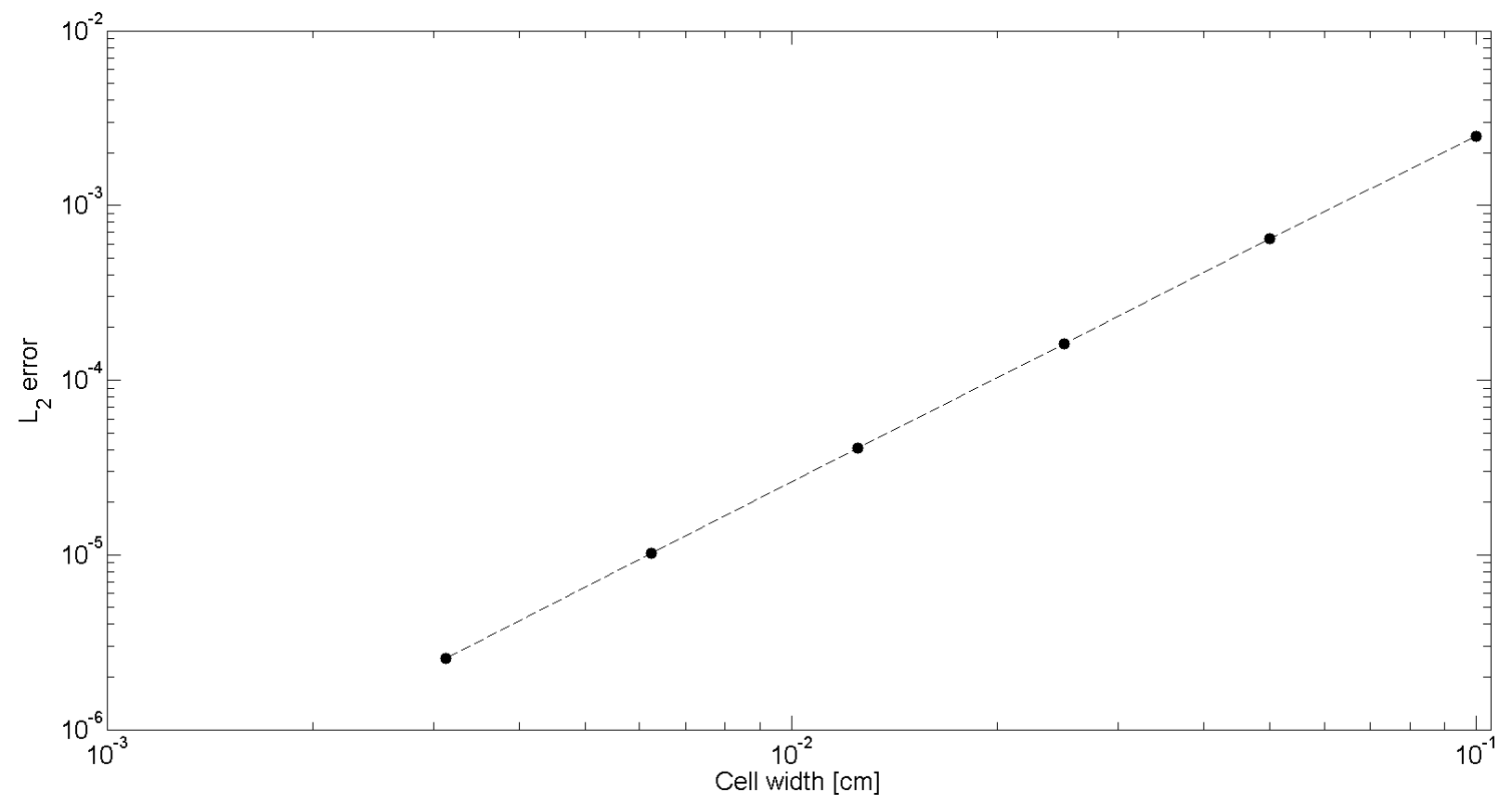

Figure 6.1: Numerical $\mathrm{L}_{2}$ error as a function of cell width for the manufactured test problem.

\subsection{Compatibility in Void}

The problem considered has two regions. The left region has a thickness of $1 \mathrm{~cm}$, is purely absorbing with an absorption cross section of $1.0 \mathrm{~cm}^{-1}$, and has as isotropic flux incident upon the outer boundary that is normalized $1 \mathrm{p} / \mathrm{s}$. The right region also has a thickness of $1 \mathrm{~cm}$, is a void, and has a vacuum condition at the outer boundary. A cell width of $0.005 \mathrm{~cm}$ is used throughout the domain in conjunction with an $\mathrm{S}_{8}$ Gauss quadrature. The solution is plotted in Fig. 6.2 along with the analytic transport solution. It can be seen that the solution is quite accurate and well-behaved at the interface between the absorber and void regions. 


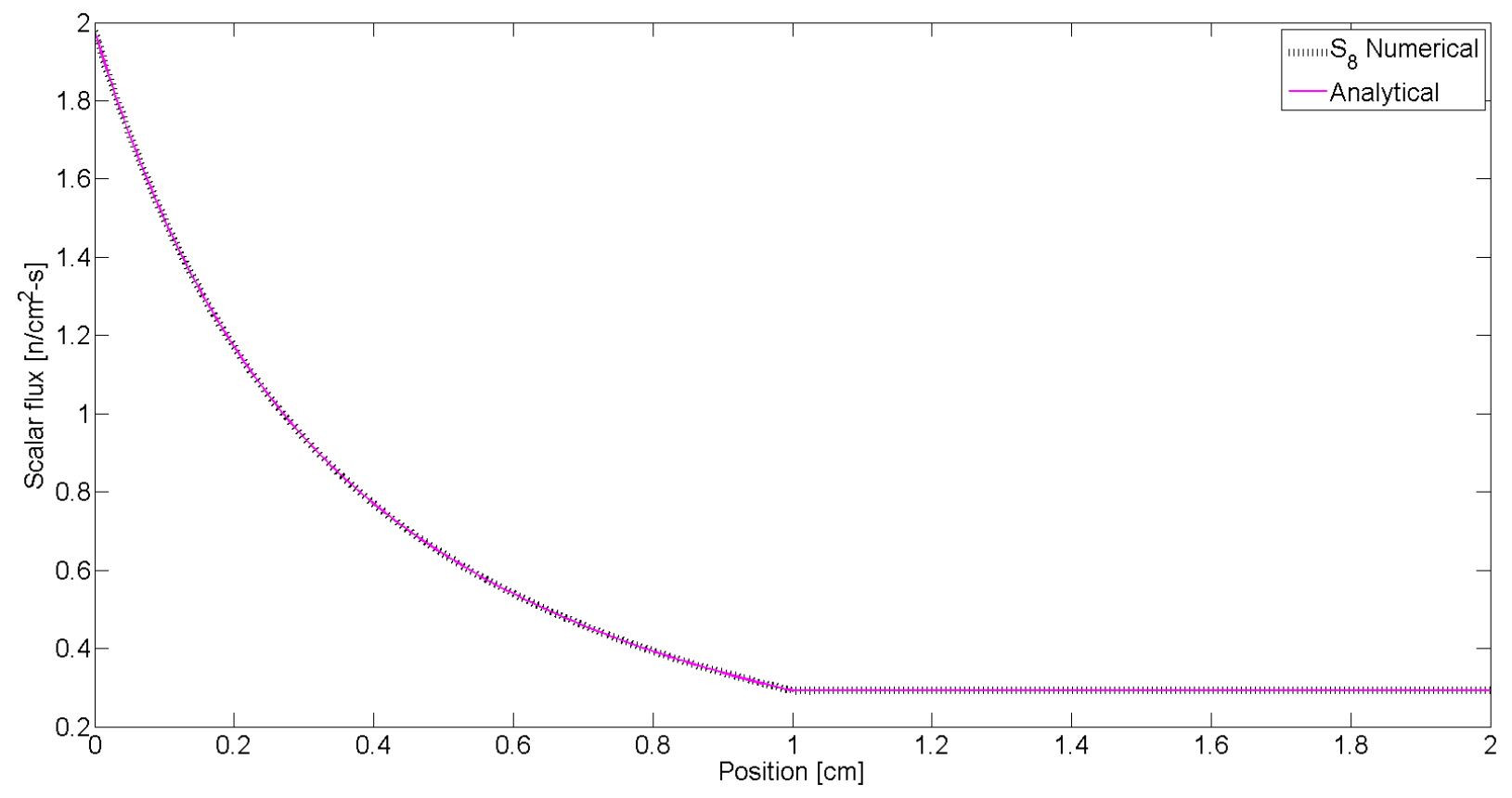

Figure 6.2: Scalar flux solution for problem with void. "Analytical" refers to the analytic transport solution.

\subsection{Spectral Radius}

We next present a set of calculations to determine the spectral radius of our method with DSA as a function of cell thickness. The test problem consists of a single region with 1000 cells of uniform thickness. Calculations are performed with cell thicknesses of $0.1 \mathrm{mfp}$ and $2^{k} \mathrm{mfp}$, where $k$ assumes all integer values between 0 and 9. There are no boundary or distributed sources, which means that the solution is identically zero. Thus the solution at each iteration step represents the error, and the norm of the solution at one iteration divided by the norm of the solution at the previous iteration represents an estimate of the spectral radius. Use of a zero source makes it possible to converge the spectral radius without concern for round-off errors that might arise after many iterations with a non-zero solution. Vacuum conditions are imposed at both boundaries, with $\sigma_{t}=\sigma_{s}=1.0 \mathrm{~cm}^{-1}$. An $\mathrm{S}_{8}$ Gauss quadrature 
is used. The scalar flux is initialized with a random number generator to ensure the presence of all frequencies. The iterations are continued until the spectral radius converges to a pointwise relative tolerance of $1 \times 10^{-4}$. The spectral radius as a function of cell thickness in plotted in Fig. 6.3. It can be seen that the spectral radius is roughly equal to 0.23 for thin cells and saturates at a value of roughly 0.5 for thick cells. While not necessarily optimal, this is nonetheless considered a good result for the numerical scheme.

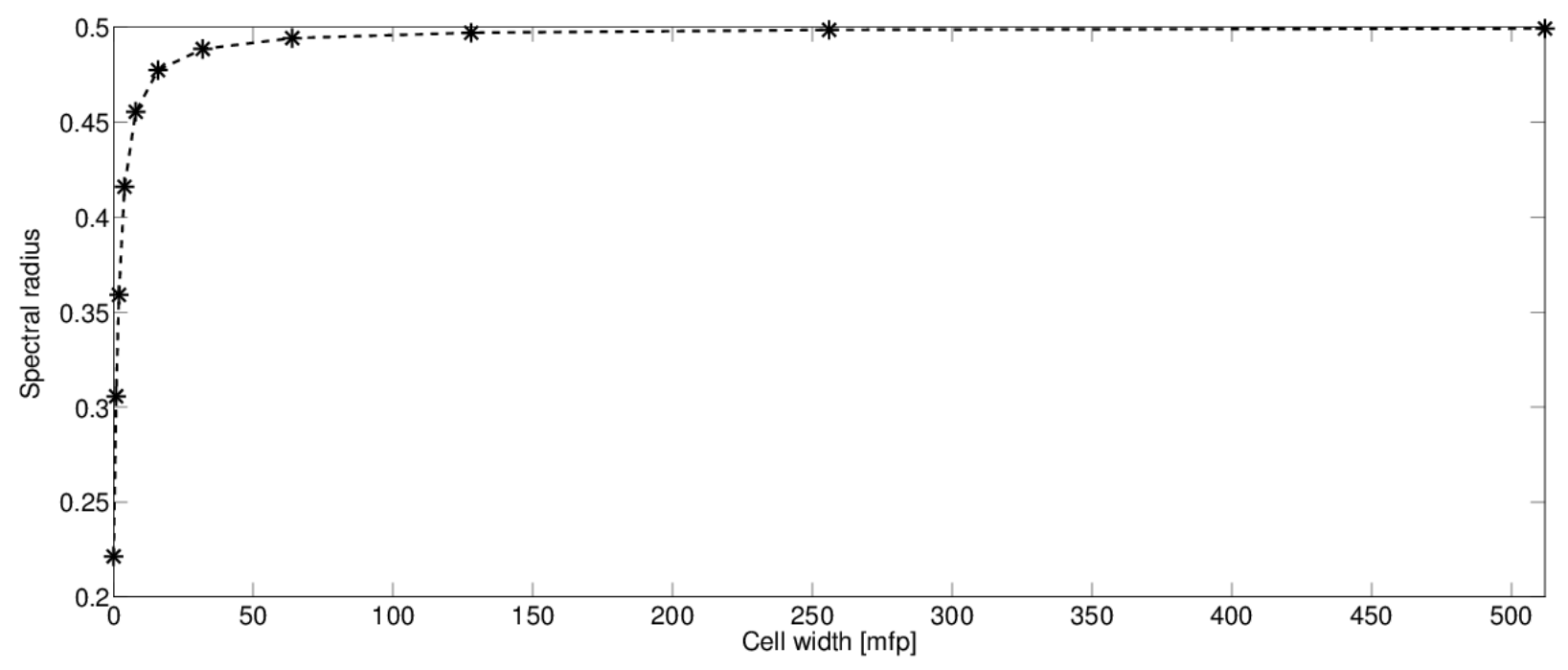

Figure 6.3: Spectral radius as a function of cell thickness.

\subsection{Rate of Convergence}

Next, we verify the rate of spatial convergence of the transport discretization. For a continuous piecewise linear finite element method the convergence rate should be second-order. We performed convergence tests using uniform meshes, but the results hold when the spatial mesh size is randomized in each region. The error associated with the numerical solution was measured using the $L_{2}$ norm: 


$$
\xi=\sqrt{\sum h\left(\phi^{\text {exact }}\left(x_{i}\right)-\phi_{i}^{\text {numerical }}\right)^{2}}
$$

The first test problem has two regions with a vacuum condition at both boundaries and a constant isotropic distributed source normalized to $1 \mathrm{p} / \mathrm{s}$ defined over the entire domain. The first region is $1 \mathrm{~cm}$ thick with the material properties $\sigma_{t}=1.0$ $\mathrm{cm}^{-1}$ and $\sigma_{a}=0.05 \mathrm{~cm}^{-1}$. The second region is $1 \mathrm{~cm}$ thick with the material properties $\sigma_{t}=0.5 \mathrm{~cm}^{-1}$ and $\sigma_{a}=0.01$. An $\mathrm{S}_{2}$ Gauss quadrature is utilized in this case in order to compare with the exact analytic solution using diffusion theory with Mark boundary conditions. The cell thickness is uniform across the domain with an initial value of $h=0.1 \mathrm{~cm}$. The cell width is decreased by a factor of 2 during each subsequent calculation. This problem converges with second-order accuracy for both vacuum and reflective boundary conditions. Numerical error data as a function of cell width are presented in Table 6.1 showing second-order spatial convergence. The second test problem features two regions with an isotropic flux incident on one boundary and a vacuum condition on the opposite boundary. The incident current is normalized to $1 \mathrm{p} / \mathrm{s}$. The errors as a function of cell width are given in Table 6.2. Once again, the numerical solution converges with second-order accuracy. The convergence rate for each test problem with a continuous source term is plotted in Fig. 6.4 showing second-order convergence. 
Table 6.1: Numerical error as a function of cell thickness for convergence test problem with an isotropic distributed source.

\begin{tabular}{ccc}
\hline Cell Width $[\mathrm{cm}]$ & $\mathrm{L}_{2}$ Error & Error Ratio \\
\hline 0.1 & $7.67 \times 10^{-4}$ & - \\
0.05 & $1.88 \times 10^{-4}$ & 4.08 \\
0.025 & $4.64 \times 10^{-5}$ & 4.05 \\
0.0125 & $1.15 \times 10^{-5}$ & 4.03 \\
0.00625 & $2.88 \times 10^{-6}$ & 3.99 \\
0.003125 & $7.19 \times 10^{-7}$ & 4.01
\end{tabular}

Table 6.2: Numerical error as a function of cell thickness for convergence test problem with an incident flux at the boundary.

\begin{tabular}{ccc}
\hline Cell Width $[\mathrm{cm}]$ & $\mathrm{L}_{2}$ Error & Error Ratio \\
\hline 0.1 & $1.31 \times 10^{-4}$ & - \\
0.05 & $3.17 \times 10^{-5}$ & 4.13 \\
0.025 & $7.79 \times 10^{-6}$ & 4.06 \\
0.0125 & $1.93 \times 10^{-6}$ & 4.04 \\
0.00625 & $4.81 \times 10^{-7}$ & 4.01 \\
0.003125 & $1.20 \times 10^{-7}$ & 4.01
\end{tabular}




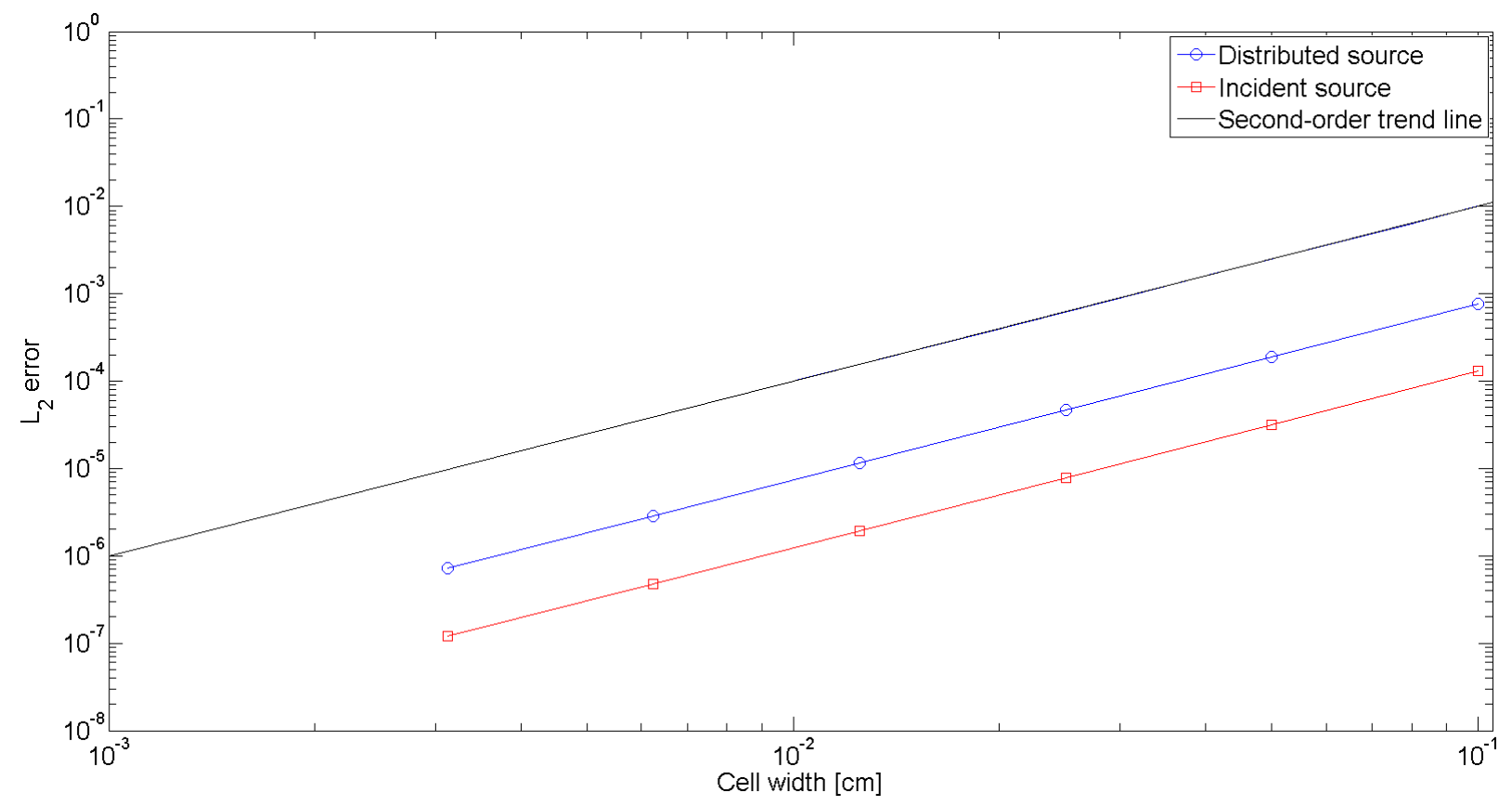

Figure 6.4: Numerical error as a function of cell thickness showing second-order spatial convergence for test problems featuring a continuous source term.

\subsection{Intermediate Diffusion Limit}

We next present results from a series of calculations that test the behavior of our scheme in the intermediate diffusion limit. The initial test problem consists of two regions, each with a thickness of $1 \mathrm{~cm}$ and and initial cell width $h=0.1 \mathrm{~cm}$ uniformly. The left region has $\sigma_{t}=10.0 \mathrm{~cm}^{-1}$ and $\sigma_{a}=0.5 \mathrm{~cm}^{-1}$, while the right region has $\sigma_{t}=5.0 \mathrm{~cm}^{-1}$ and $\sigma_{a}=0.1 \mathrm{~cm}^{-1}$. There is a constant isotropic distributed source in both regions normalized to $1 \mathrm{p} / \mathrm{s}$ and vacuum boundary conditions on each side of 
the slab. The asymptotic scaling associated with the intermediate limit is as follows:

$$
\begin{aligned}
\sigma_{t} & \rightarrow \frac{1}{\epsilon} \sigma_{t}, \\
\sigma_{a} & \rightarrow \epsilon \sigma_{a}, \\
q & \rightarrow \epsilon q, \\
h & \rightarrow \epsilon h,
\end{aligned}
$$

where $\epsilon$ denotes the asymptotic scaling parameter and $h$ denotes the cell width. Note that the cell thickness in $m f p$ is equal to $\sigma_{t} h$ and does not depend upon the value of $\epsilon$ in the intermediate limit. Also, the thickness of each cell in the intermediate limit is on the order of $\sim 1 \mathrm{mfp}$. This is the origin of the term "intermediate." In the "thin" limit, the cell thickness in $m f p$ goes to zero with $\epsilon$. All consistent spatial discretization schemes converge to the asymptotic diffusion solution in the thin limit. The initial problem described corresponds to $\epsilon=1.0$. We perform calculations for $\epsilon=1.0,0.1,0.05$, and 0.01 while using an $S_{16}$ Gauss quadrature. The corresponding scalar flux solutions for each $\epsilon$ value are compared with the analytic asymptotic diffusion solution, and the rate of convergence is studied in detail in the intermediate diffusion limit. Table Table 6.3 shows the $L_{2}$ numerical error as a function of $\epsilon$. The numerical solution converges to the analytic solution as $\epsilon \rightarrow 0$, implying that our transport discretization preserves the intermediate diffusion limit, which can be important for accuracy in $k$-eigenvalue calculations. The convergence test shown in Fig. 6.5 demonstrates first-order accuracy for the scheme in the intermediate diffusion limit. This is expected because the leading order asymptotic solution is being approached by the transport solution with first-order error in $\epsilon$. 
Table 6.3: Numerical error as a function of $\epsilon$ in the intermediate diffusion limit.

\begin{tabular}{cc}
\hline$\epsilon$ & $\mathrm{L}_{2}$ Error \\
\hline 1.0 & 0.3847 \\
0.1 & 0.0409 \\
0.05 & 0.0205 \\
0.01 & 0.0041 \\
\hline
\end{tabular}

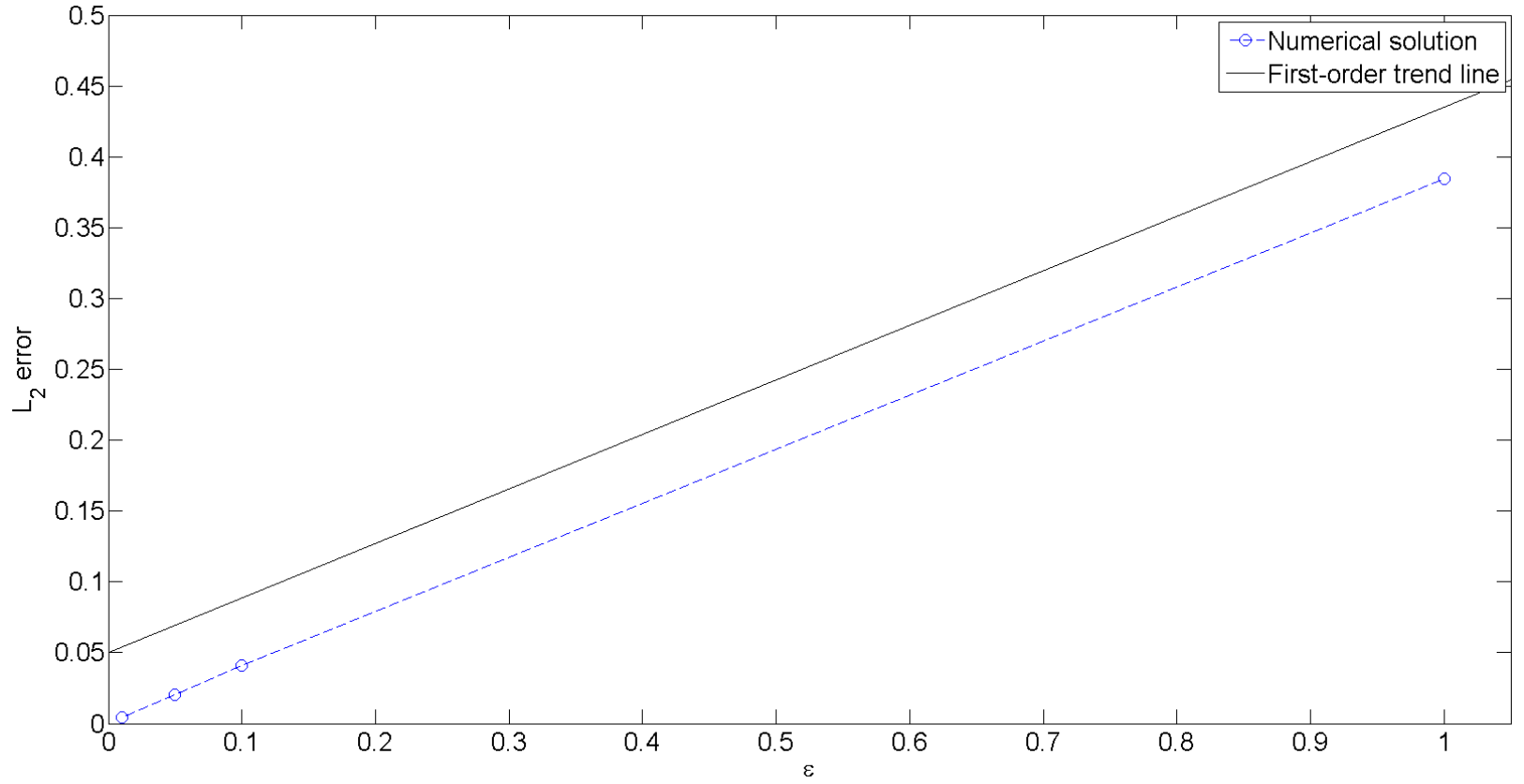

Figure 6.5: Numerical error as a function of $\epsilon$ in the intermediate diffusion limit showing first-order accuracy. 


\subsection{Thick Diffusion Limit}

Finally, we present results from a series of calculations to test the behavior of our scheme in the thick diffusion limit. For the thick limit, the asymptotic scaling is nearly the same as the intermediate limit scaling, with the sole exception that cell width $h$ is not scaled by $\epsilon$ in this case:

$$
\begin{gathered}
\sigma_{t} \rightarrow \frac{1}{\epsilon} \sigma_{t}, \\
\sigma_{a} \rightarrow \epsilon \sigma_{a}, \\
q \rightarrow \epsilon q, \\
h \rightarrow h .
\end{gathered}
$$

Note that the cell thickness in $m f p$ becomes infinite as $\epsilon$ is decreased. This is the origin of the term "thick." Similar to the intermediate diffusion limit problem, the thick limit test problem features two $1 \mathrm{~cm}$ thick regions containing a constant isotropic distributed source normalized to $1 \mathrm{p} / \mathrm{s}$ with vacuum boundary conditions on each side of the slab. In this case the material properties are made to be highly diffusive, where the left region has $\sigma_{t}=10,000 \mathrm{~cm}^{-1}$ and $\sigma_{a}=5.0 \times 10^{-4} \mathrm{~cm}^{-1}$, while the right region has $\sigma_{t}=5,000 \mathrm{~cm}^{-1}$ and $\sigma_{a}=1.0 \times 10^{-4} \mathrm{~cm}^{-1}$. We perform thick limit scaling calculations for $\epsilon=1.0,0.1,0.05$, and 0.01 with an $S_{16}$ Gauss quadrature. The corresponding scalar flux solutions with uniform cell width set to $h=0.1 \mathrm{~cm}$ are compared with the analytic asymptotic diffusion solution in Fig. 6.6. The numerical solution is not expected to precisely converge for decreasing $\epsilon$, since the truncation error due to the spatial discretization does not decrease while cell width $h$ is held constant. Nonetheless, it can be seen that the agreement between 
the numerical and analytic solutions in Fig. 6.6 is reasonable. Such agreement is generally accepted as an indication that a scheme preserves the thick diffusion limit; however, we perform another series of calculations to gather additional evidence in this regard.

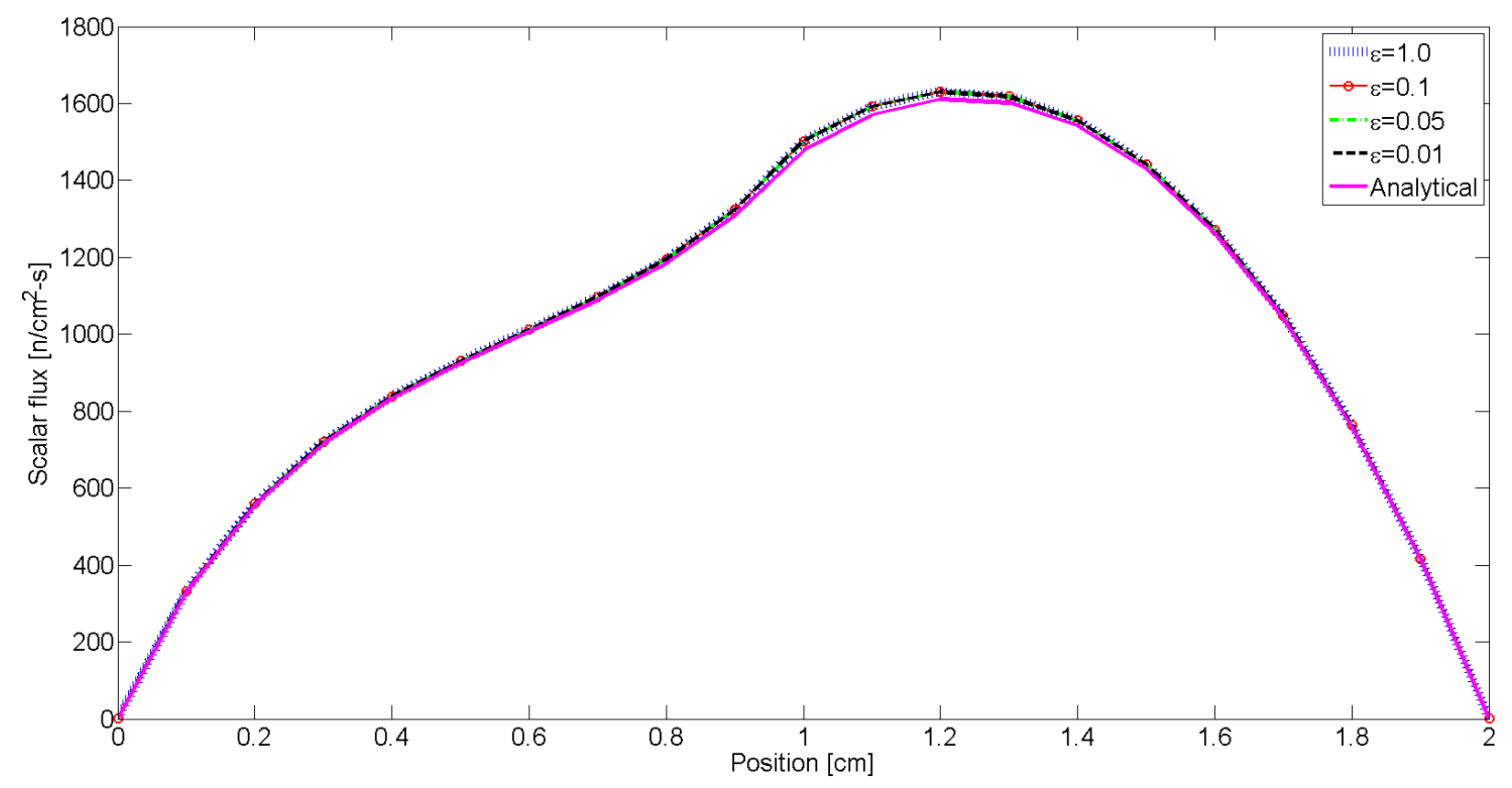

Figure 6.6: Scalar flux solutions as a function of $\epsilon$ in the thick diffusion limit. "Analytical" refers to the analytic asymptotic diffusion solution.

More specifically, we perform a set of calculations using the intermediate limit scaling, but note that the thickness of each spatial cell is set to a very large number of mean-free-paths. The problem characteristics for this test case are the same as described above for the thick limit problem. The cell width is uniform and set to $h=0.1 \mathrm{~cm}$ initially; therefore, the cell width with regard to $m f p$ is constant at 1,000 $\mathrm{mfp}$ in the first region and $500 \mathrm{mfp}$ in the second region for varying $\epsilon=$ 1.0, $0.1,0.05$, and 0.01 using the intermediate limit scaling. The $L_{2}$ numerical error 
relative to the analytical asymptotic diffusion solution is found at each $\epsilon$ value, and the results are given in Table 6.4 and plotted in Fig. 6.7. It is clear from Fig. 6.7 that the numerical error is reduced approximately first-order in $\epsilon$ for the thick limit test problem. The overall behavior observed indicates that our transport discretization preserves the thick diffusion limit.

Table 6.4: Numerical error as a function of $\epsilon$ for the intermediate limit test with very thick cells.

\begin{tabular}{cc}
\hline$\epsilon$ & $\mathrm{L}_{2}$ Error \\
\hline 1.0 & 17.0977 \\
0.1 & 1.4509 \\
0.05 & 0.7203 \\
0.01 & 0.1671
\end{tabular}




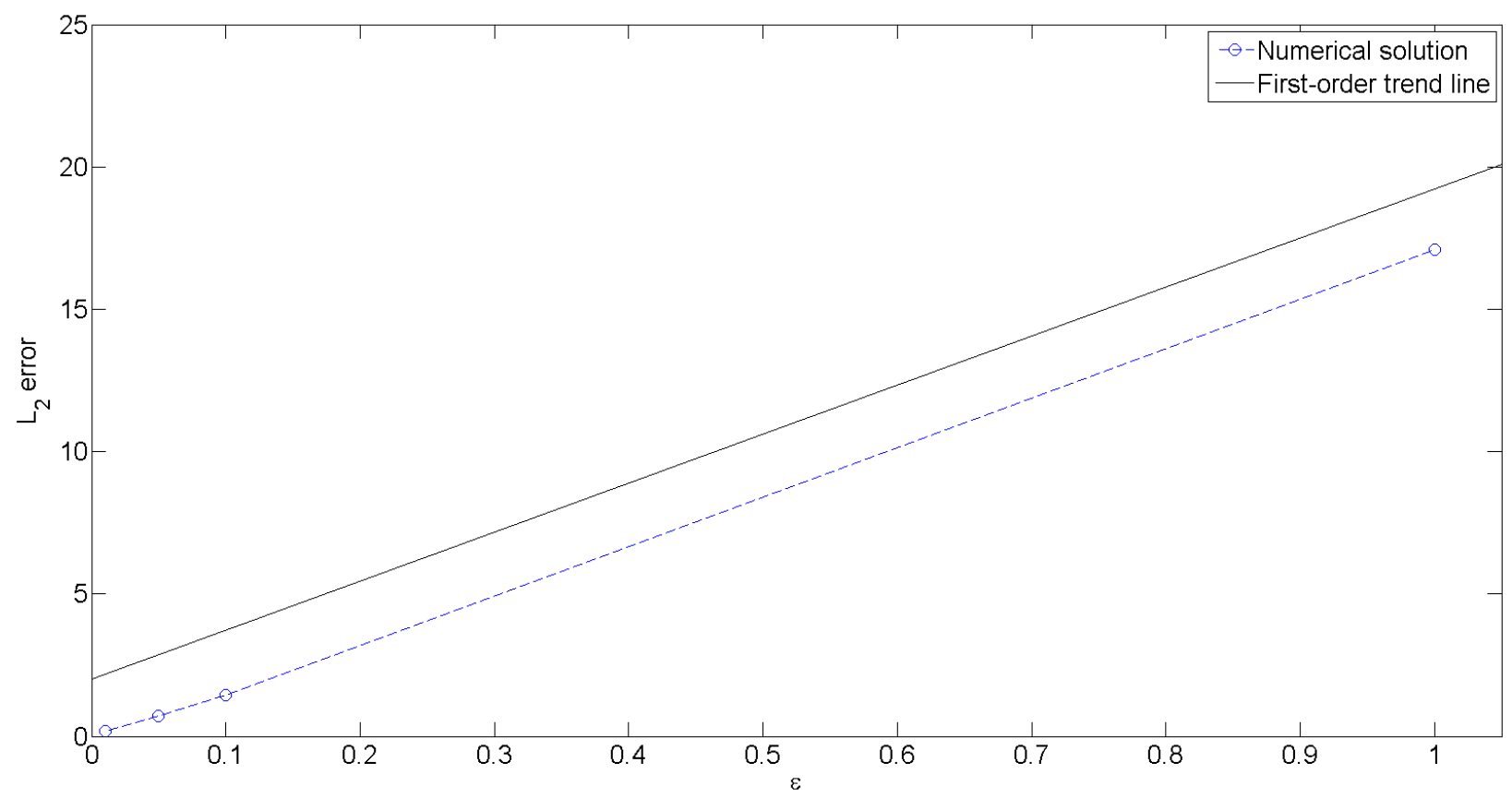

Figure 6.7: Numerical error as a function of $\epsilon$ for the intermediate limit test with very thick cells showing approximately first-order accuracy.

\subsection{Unresolved Boundary Layers}

It is beneficial to evaluate the behavior of our least-squares transport scheme in the thick diffusion limit with an unresolved boundary layer. The asymptotic solution is non-diffusive in a boundary layer. If the layer is not resolved, the best one can hope for in the boundary cell containing the layer is to obtain the correct diffusive solution in the cell interior extrapolated through the layer to the boundary. We expect our discretization to perform poorly in this situation, given the fact that Dirichlet boundary conditions are used. Good performance in a boundary layer problem requires that the interor solution be able to "disconnect" from the incident transport flux at the boundary, yet this cannot take place with our continuous discretization.

To test our discretization we consider two test problems in which the thick-limit 
scaling factors defined in Eqs. (6.6a) through (6.6d) are imposed. These two problems differ only with respect to the incident angular flux distribution. For the first test case, an isotropic incident flux normalized to an incoming current of $1 \mathrm{p} / \mathrm{s}$ is imposed on the left boundary. There is no boundary layer in this case, so we expect good results. In the second case, a highly anisotropic incident flux is imposed on the left boundary. The flux is non-zero only in the direction closest to $\mu=1$ and the incident current is normalized to $1 \mathrm{p} / \mathrm{s}$. An incident anisotropic flux always generates a boundary layer. For both problems, the cell width is uniform with $h=0.05 \mathrm{~cm}$. The left region is $1 \mathrm{~cm}$ thick with $\sigma_{t}=1.0 \mathrm{~cm}^{-1}$ and $\sigma_{a}=1.0 \mathrm{~cm}^{-1}$ initially. The right region has a thickness of $1 \mathrm{~cm}$ with $\sigma_{t}=0.5 \mathrm{~cm}^{-1}$ and $\sigma_{a}=0.5 \mathrm{~cm}^{-1}$ initially, and has a vacuum boundary condition on the right side of the slab. Calculations are performed for the thick limit for both test problems with $\epsilon=1,0.1,0.01$, and $1 \times 10^{-4}$ to demonstrate the behavior of the numeric scheme with no boundary layer and with an unresolved boundary layer. The flux solutions for the first case are shown in Fig. 6.8. The exact asymptotic scalar flux solution takes on a value of 4.0 $\frac{n}{\mathrm{~cm}^{2}-s}$ at the left bouundary and zero at the right boundary. The numerical solution shows good agreement with these values and is accurate as expected.

The flux solutions for the second case are shown in Fig. 6.9. Assuming a normallyincident angular flux, the exact asymptotic scalar flux solution takes on a value of 5.0 $\frac{n}{c^{2}-s}$ at the left boundary and zero at the right boundary. Furthermore, the exact solution should monotonically decrease from the inflow boundary to the vacuum boundary. The numerical solutions obtained with our transport discretization are both quantitatively and qualitatively inaccurate near the source boundary. Because Dirichlet boundary conditions are used, we know that our transport disretization cannot be expected to produce an accurate result with an unresolved boundary layer, and our results confirm that expectation. Good results require that the boundary 
layer be resolved. In particular, one must use cells small with respect to a mean-freepath within the boundary layer. Optically-thick cells should be used only within the diffusive region. In principle, one could obtain accuracy with an unresolved boundary layer by using a penalty term to weakly enforce the boundary condition. However, this would do nothing for interior boundary layers. Accuracy for unresolved interior boundary layers would require a fundamentally different spatial discretization.

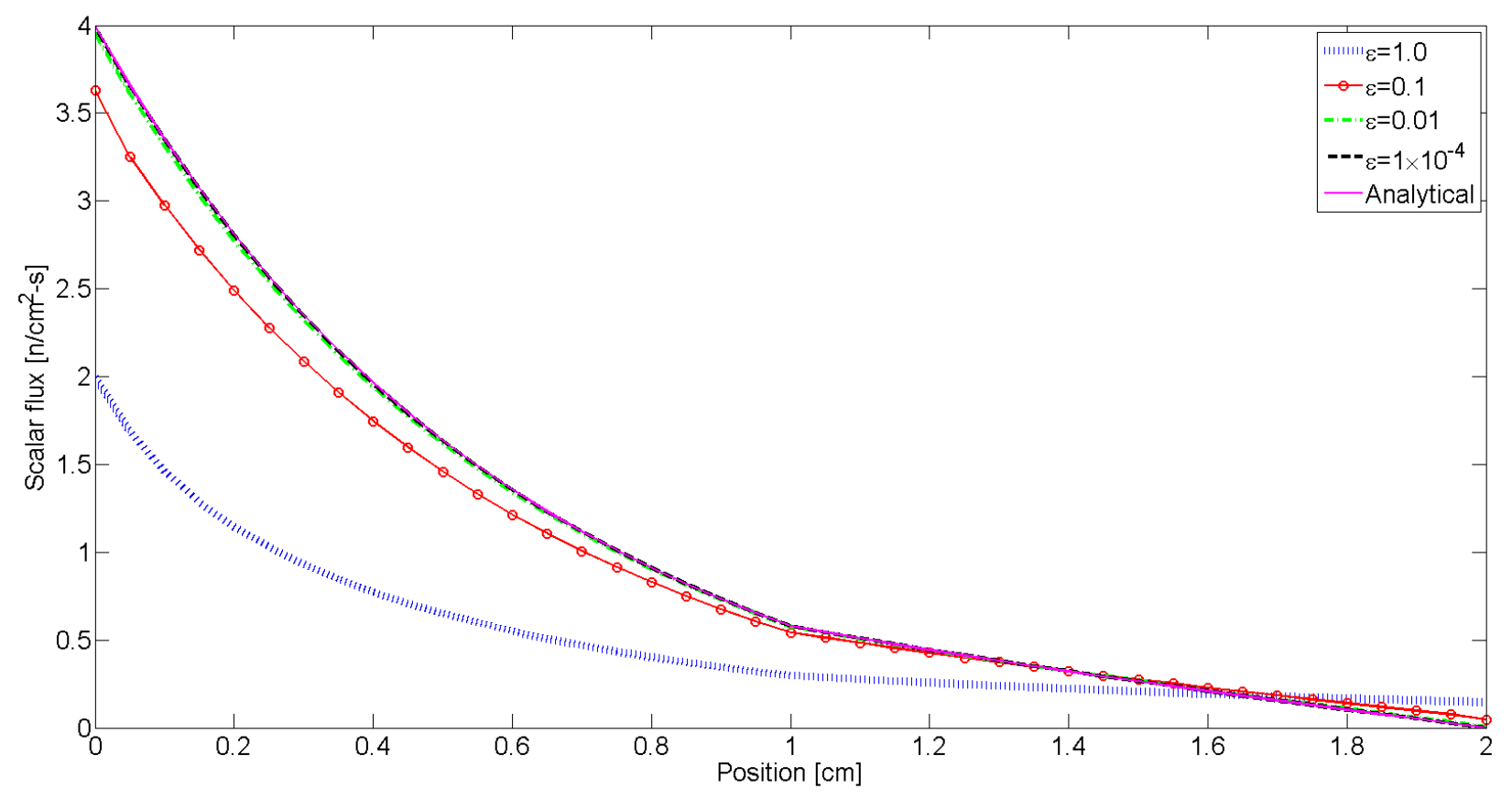

Figure 6.8: Scalar flux solutions for decreasing $\epsilon$ in the thick limit with an incident isotropic boundary flux. "Analytical" refers to the analytic asymptotic diffusion solution. 


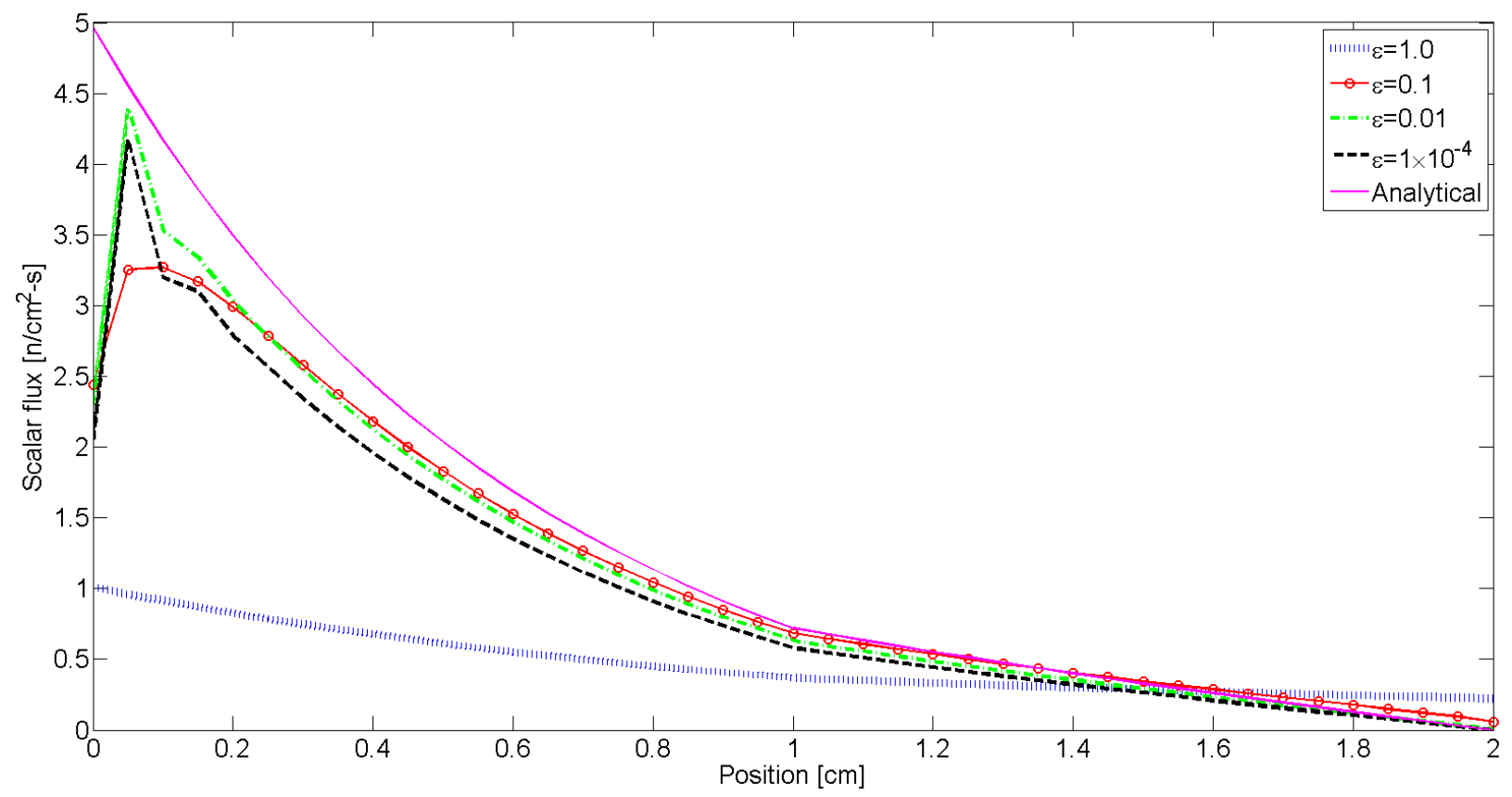

Figure 6.9: Scalar flux solutions for decreasing $\epsilon$ in the thick limit with a unidirectional source normal to the slab showing incorrect behavior within the boundary layer. "Analytical" refers to the analytic asymptotic diffusion solution.

\subsection{Interior Boundary Layers}

In this section we consider an interior boundary layer. Such boundary layers can arise for any problem with a combination of transport and diffusive regions. We define a test problem for an interior boundary layer simply by inserting a void region with a thickness of $1 \mathrm{~cm}$ to the left of the unresolved boundary layer problem defined previously and imposing the same anisotropic flux incident on the void. The boundary layer then occurs at the interface between the purely absorbing and first diffusive region. The numerical solutions under the thick limit analysis for this test problem with $\epsilon=1,0.1,0.01$, and $1 \times 10^{-4}$ are shown in Fig. 6.10. The solution is not correct as it should be $5.0 \frac{n}{\mathrm{~cm}^{2}-\mathrm{s}}$ at the interface between the void and the first diffusive region and zero at the right boundary. It should also be constant in the 
void region. This poor performance with an unresolved interior boundary layer is expected since our discretization is continuous. Our results indicate that boundary layers must be resolved in order to effectively use our transport discretization whether they occur at outer boundaries or at internal material interfaces.

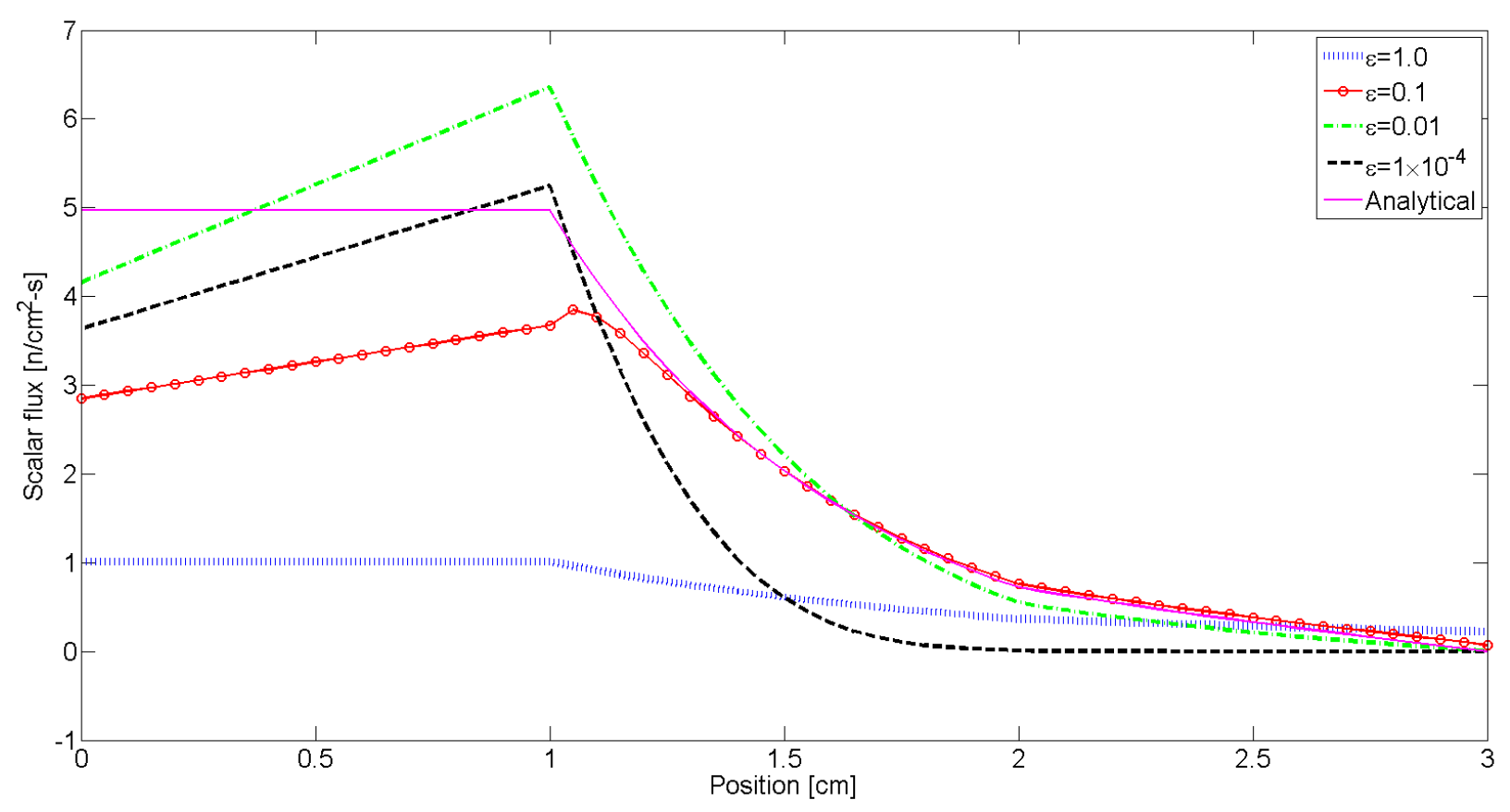

Figure 6.10: Scalar flux solutions for decreasing $\epsilon$ in the thick limit with an unresolved interior boundary layer. "Analytical" refers to the analytic asymptotic diffusion solution given void entry with known scalar flux value. 


\section{CONCLUSION}

We have developed a least-squares form of the transport equation and an associated discretized equation that can be used in voids and near-voids, can be efficiently solved using source iteration with diffusion-synthetic acceleration, and possesses both the intermediate and thick diffusion limits. If used for highly diffusive problems, all boundary layers must be spatially resolved. The thick diffusion limit is sometimes relevant for radiative transfer calculations, but it is generally not relevant for reactor neutronics calculations. This scheme clearly represents a valuable alternative to other second-order forms of the transport equation, particularly if voids or near-voids are present in problems. In the future we intend to investigate this scheme in 3-D and collaborate with others to develop a multigrid scheme for solving the source iteration equations associated with our scheme. It appears that there are theoretical indications that our equation may have certain advantages for the development of a multigrid algorithm relative to the standard least-squares form of the transport equation [11]. Finally, future work involves the implementation of a low-order/high-order scheme based upon our least-squares $\mathrm{S}_{n}$ equations for reactor physics calculations using the MOOSE framwork at Idaho National Laboratory [12]. Additionally, results should be shown for realistic near-void problems that demonstrate highly anomalous spatial convergence properties for the even-parity $\mathrm{S}_{n}$ equations and the $\mathrm{SP}_{n}$ equations, but express good spatial convergence properties for our least-squares $\mathrm{S}_{n}$ equations. 


\section{REFERENCES}

[1] E. E. Lewis and W. F. Miller Jr., Computational Methods of Neutron Transport, American Nuclear Society, Lagrange Park, IL (1993).

[2] J. E. Morel and J. M. McGhee, "A Self-Adjoint Angular Flux Equation," Nuclear Science and Engineering, 132, 312-325 (1999).

[3] R.T. Ackroyd, J.G. Issa, N.S. Riyait, "Treatment of Voids in Finite Element Transport Methods," Progress in Nuclear Energy, 18, 85-89 (1986).

[4] Cliff Drum, Wesley Fan, Andrew Bielen, Jeffrey Chenhall, "Least-Squares Finite-Element Algorithms in the SCEPTRE Radiation Transport Code," International Conference on Mathematics and Computational Methods Applied to Nuclear Science and Engineering (MEC 2011), Rio de Janeiro, RJ, Brazil, May 8-12, 2011, on CD-ROM, Latin American Section (LAS) / American Nuclear Society (ANS) ISBN 978-85-63688-00-2 (2011).

[5] T.A. Manteuffel and K.J. Ressel, "Least-Squares Finite-Element Solution of the Neutron Transport Equation in Diffusive Regimes," SIAM Journal on Numerical Analysis, 35(2), 806-835 (2002).

[6] Edward W. Larsen, J. E. Morel, Warren F. Miller Jr., "Asymptotic Solutions of Numerical Transport Problems in Optically Thick, Diffusive Regimes," Journal of Computational Physics, 69, 283-324 (1987).

[7] W. Daryl Hawkins, Timmie Smith, Michael P. Adams, Lawrence Rauchwerger, Nancy Amato, Marvin Adams, "Efficient Massively-Parallel Transport Sweeps," Transactions of the American Nuclear Society, 107, 477-481 (2012). 
[8] E. W. Larsen and J. B. Keller, "Asymptotic Solution of Neutron Transport Problems for Small Mean Free Paths," Journal of Mathematical Physics, 15, 75-81 (1974).

[9] F. Malvagi and G. C. Pomraning, "Initial and Boundary Conditions for Diffusive Linear Transport Problems," Journal of Mathematical Physics, 32(3), 805-820 (1991).

[10] MATLAB 2013a. Natick, Massachusetts: The MathWorks Inc.

[11] Personal communication, Thomas Manteuffel, University of Colorado, Boulder.

[12] D. Gaston, C. Newman, G. Hansen, D. Lebrun-Grandie, "MOOSE: A Parallel Computational Framework for Coupled Systems of Nonlinear Equations," Nuclear Engineering and Design, 239, 1768-1778 (2009). 